Document downloaded from:

http://hdl.handle.net/10251/99442

This paper must be cited as:

Rus, G.; Palma, R.; Pérez-Aparicio, JL. (2012). Experimental design of dynamic modelbased damage identification in piezoelectric ceramics. Mechanical Systems and Signal Processing. 26:268-293. doi:10.1016/j.ymssp.2011.06.023

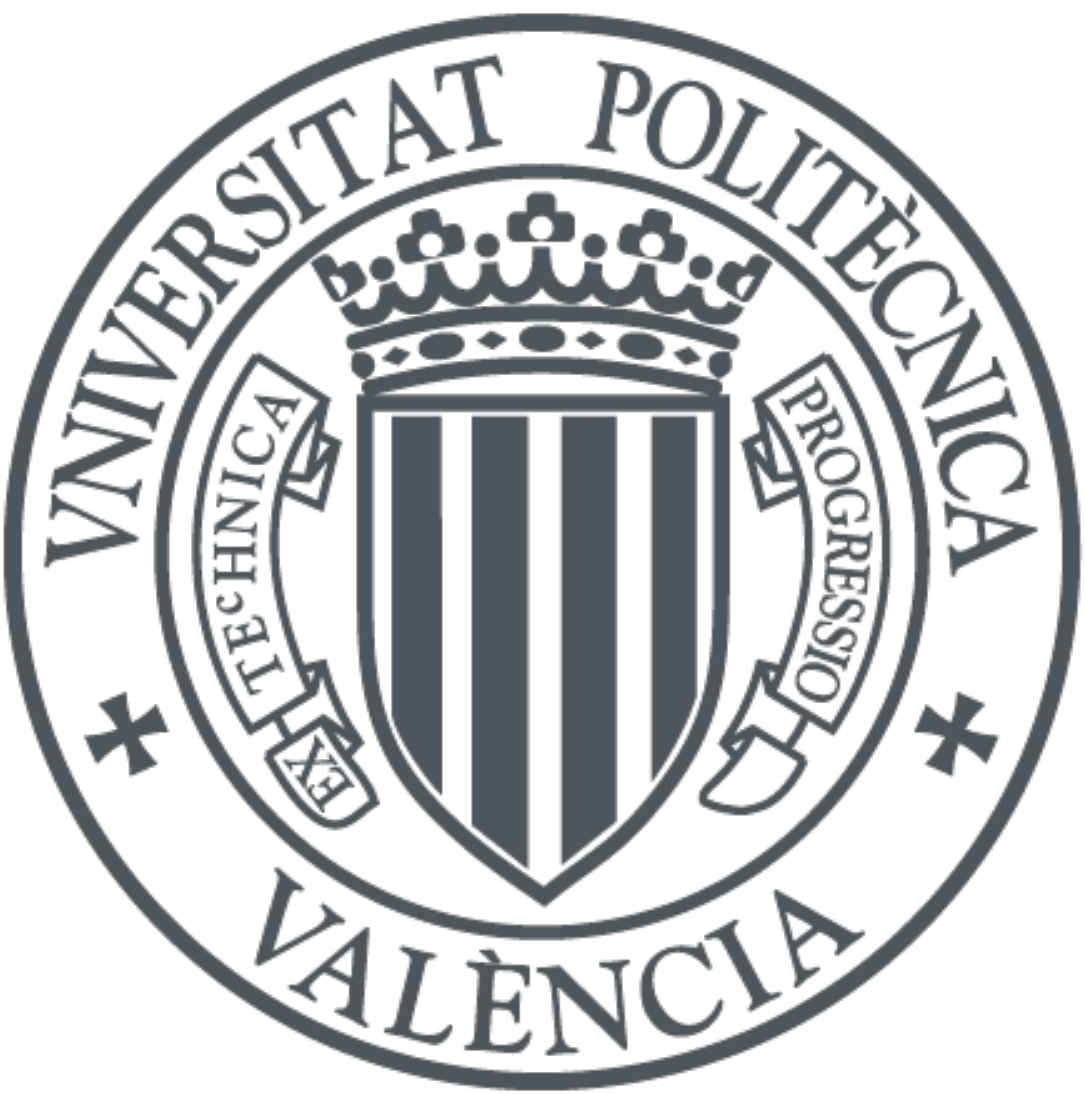

The final publication is available at

http://doi.org/10.1016/j.ymssp.2011.06.023

Copyright ACADEMIC PRESS LTD- ELSEVIER SCIENCE LTD

Additional Information 
Elsevier Editorial System(tm) for Journal of the Mechanics and Physics of Solids Manuscript Draft

Manuscript Number:

Title: Experimental design of dynamic model-based damage identification in piezoelectric ceramics

Article Type: Research Paper

Corresponding Author: Dr. Guillermo Rus, Ph.D.

Corresponding Author's Institution: University of Granada

First Author: Guillermo Rus, Ph.D.

Order of Authors: Guillermo Rus, Ph.D.; Roberto Palma; José Luis Pérez-Aparicio, Ph.D.

Abstract: A model-based inverse problem strategy is proposed for damage characterization, starting from the electromechanical response measurement as input data, and incorporating a numerical model that simulates the piezoelectric response. Furthermore, a sensitivity analysis is developed to provide a rational basis to correctly design the excitation/measurement system. The model-based inverse problem is solved by minimizing a cost functional using genetic algorithms. The cost functional or discrepancy is defined as the L-2 norm of the difference between experimental and simulated measurements. The latter are obtained by solving the forward problem, using a novel 2-D dynamic piezoelectric finite element. The effect of measurement noise and model uncertainties is studied in detail through a sensitivity analysis, where a sensitivity factor is defined and implemented. The proposed inverse problem strategy reconstructs the defect characteristics with sufficient precision, under realistic levels of noise.

Suggested Reviewers: Nader Saffari Ph.D.

Senior Reader, Mechanical Engineering, University College London

n.saffari@ucl.ac.uk

Renowned expert in characterization of complex materials with dynamic measurements.

Sang Youl Lee Ph.D.

Associate Professor, Civil Engineering, Joongbu University

leesangyoul@hanmail.net

Renowned expert in inverse problems and finite elements for damage characterization by dynamic measurements.

Barbara Kaltenbacher Ph.D.

Professor, INSTITUT FÜR MATHEMATIK UND WISSENSCHAFTLICHES RECHNEN, University of Graz barbara.kaltenbacher@uni-graz.at

Expert in characterization of properties in piezoelectric materials.

Opposed Reviewers: 


\title{
Experimental design of dynamic model-based damage identification in piezoelectric ceramics
}

\author{
G. Rus ${ }^{\mathrm{a}}$, R. Palma ${ }^{\mathrm{a}}$, J.L. Pérez-Aparicio ${ }^{\mathrm{b}}$ \\ ${ }^{a}$ Structural Mechanics and Hydraulic Engineering, University of Granada, Spain \\ ${ }^{b}$ Mecánica de Medios Continuos y Teoría de Estructuras, Universidad Politécnica de \\ Valencia, Spain
}

\begin{abstract}
A model-based inverse problem strategy is proposed for damage characterization, starting from the electromechanical response measurement as input data, and incorporating a numerical model that simulates the piezoelectric response. Furthermore, a sensitivity analysis is developed to provide a rational basis to correctly design the excitation/measurement system. The model-based inverse problem is solved by minimizing a cost functional using genetic algorithms. The cost functional or discrepancy is defined as the $L-2$ norm of the difference between experimental and simulated measurements. The latter are obtained by solving the forward problem, using a novel 2-D dynamic piezoelectric finite element. The effect of measurement noise and model uncertainties is studied in detail through a sensitivity analysis, where a sensitivity factor is defined and implemented. The proposed inverse problem strategy reconstructs the defect characteristics with sufficient precision, under realistic levels of noise.
\end{abstract}

Keywords: Inverse problem, damage identification, piezoelectrics, finite element method, genetic algorithms

\section{Introduction}

Piezoelectric ceramics have recently become one of the most used materials for all types of electromechanical systems. However, the presence of defects can prevent them from safely fulfilling their function. Many numerical, analytical and experimental works have recently being developed to understand the piezoelectric behavior in the presence of damage, but very few are aimed at locating these defects. 
Kuna (2010) and Schneider (2007) review, respectively, analytical and experimental studies on piezoelectric materials in the presence of defects and conclude that defects strongly alter the electric and mechanical fields, inducing high stress and electric fields concentrations around the defect.

In order to simulate the basic behavior of piezoelectric ceramics from a numerical point of view, two alternative Finite Element Method (FEM) formulations emerged: scalar, Allik and Hughes (1970), and vectorial, Landis (2002). The first uses four degrees of freedom per node (three displacement and the scalar potential or voltage), while the second uses six (three displacements and three for the vector potential). For the solution of the Inverse Problem (IP), the scalar is better than the vectorial formulation, since in the laboratory it is easier to measure voltages than vector potentials.

The identification IP has been recently applied to evaluate the elastic, dielectric and coupling properties of piezoelectric ceramics. For example, Kaltenbacher et al. (2006) defined a Cost Functional (CF) as the difference between electric impedances measured in laboratory and those obtained solving the forward problem by FEM. Also, Ruíz et al. (2004) used a similar CF which was minimized using Genetic Algorithms (GA).

Also, the characterization IP was applied to locate defects in a PZT-4 plate under the plain strain assumption in Rus et al. (2009) and Palma et al. (2009). In these works, the CF was defined as the discrepancy between two measurements. The first was numerical, obtained by solving a forward problem by the FEM developed in Pérez-Aparicio et al. (2007). The second was experimental, simulated by adding stochastic noise from a Gaussian function to the first. Note that real experiments were avoided in order to carry out a real analytical Sensitivity Analysis (SA). The analysis concluded that the IP solution strongly depends on the noise level on the experimental measurement. This conclusion was also reached by Tarantola (2005), where the inversion theory under a probabilistic formulation was examined to explain the robustness of the inversion. Finally, in Rus et al. (2009) the CF was minimized by GA, an heuristic optimization technique from Goldberg, D. E. (1989), and by BFGS, a gradient-based algorithm, see Dennis, J.E. and Schnabel R.B. (1983). Both algorithms were compared, concluding that GA shown a more robust convergence.

In the present work, a model-based inverse problem strategy is proposed for damage characterization, starting from Palma et al. (2009) and considering dynamical responses. To this end, a novel 2-D dynamic piezoelectric finite element based on scalar formulation is developed and implemented in 
the computer FEM code FEAP (see Taylor (2003)). The CF is minimized using GA, since this technique requires a significantly smaller (compared to gradient-based methods) amount of data for complex problems, while attaining global convergence. Another goal of this work is to find which excitations and measurements provide the optimal monitoring setup, so that the IP solution yields a satisfactory damage characterization under realistic levels of error. Note that the final error is assumed to be a combination of the errors in: i) measurements (noise), ii) uncertainties in the physical properties and in the geometrical parameters of the model (model uncertainties), iii) the FEM and iv) the GA computations. For the mentioned setup, the SA of the IP is computed with a Taylor expansion and with the developments of Palma et al. (2009), that yield an expression for the IP sensitivities with respect to model uncertainties. The last two formulations are combined to find the relationships between model uncertainties and measurements on one side, and IP solutions on the other. This relationships will allow to find the variables that influence in a higher degree on the final error level.

Section 2 presents the piezoelectric governing equations and the FEM used to solve them, and subsequently the IP approach. Section 3 analyzes the possible monitoring configurations and the IP results, while Section 4 summarizes the observations and the obtained conclusions.

\section{Methodology}

The problem of nondestructive damage characterization is solved by a model-based IP approach that consists of two steps: i) excitation of the system applying mechanical or electrical loads, and ii) measurement of the response (displacements or voltage). The issues that play a role in the IP are summarized in Figure 1, and are explained in detail in this section.

\subsection{Forward problem}

The system is a specimen of PZT -4 ceramic of dimensions $L_{x}=60 \times L_{z}=$ $60[\mathrm{~mm}]$ and arbitrary thickness, which is modeled by a plain strain FEM formulation. In order to generate scalable results, the remaining dimensions (damage characteristics) are set relatively to the former, therefore they are non-dimensional and generic.

Piezoelectric equations relate displacement $\mathbf{u}=(u, v)$ and voltage $\phi$, strain $\mathbf{S}$ and field $\mathbf{E}$, stress $\mathbf{T}$ and induction $\mathbf{D}$ for the mechanical and electric energies respectively, all of them in a coupled manner. The standard and 


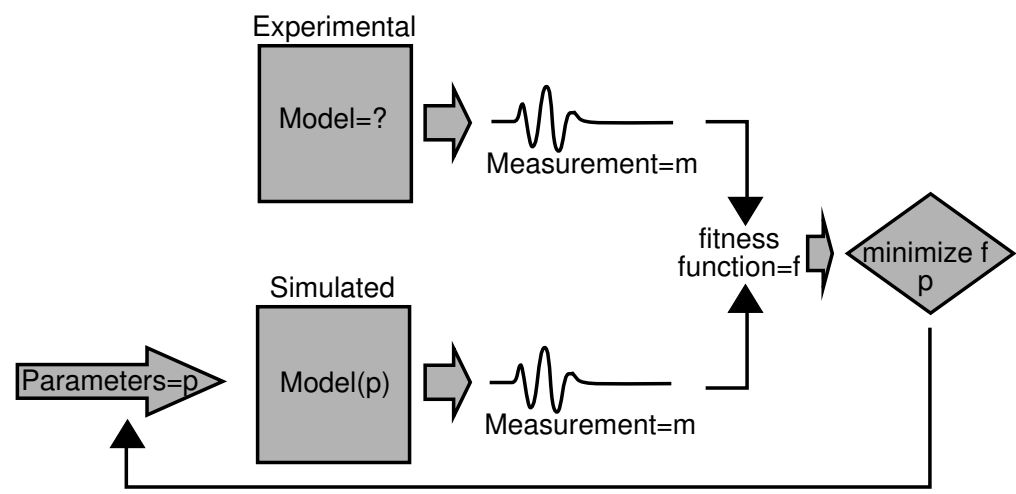

Figure 1: Issues scheme for the solution of the model-based Inverse Problem

uncoupled equilibrium equations are the Cauchy and Gauss' law,

$$
\nabla \cdot \mathbf{T}+\mathbf{f}=\rho \ddot{\mathbf{u}}, \quad \nabla \cdot \mathbf{D}=\rho_{\Omega}
$$

where $\mathbf{f}$ are the external mechanical forces, $\rho$ is the mass density and $\rho_{\Omega}$ are the volume density of electric charges. The also standard compatibility equations: small deformation and Faraday's law $\nabla \times \mathbf{E}=\mathbf{0}$,

$$
\mathbf{S}=\nabla^{s} \mathbf{u}, \quad \mathbf{E}=-\nabla \phi
$$

This set is well-posed if the proper Neumann and Dirichlet boundary conditions are prescribed,

$$
\left\{\begin{array}{c}
\mathbf{T} \cdot \mathbf{n}=\mathbf{t} \\
\mathbf{u}=\overline{\mathbf{u}}
\end{array}, \quad\left\{\begin{array}{c}
\mathbf{D} \cdot \mathbf{n}=\rho_{\Gamma} \\
\phi=\bar{\phi}
\end{array}\right.\right.
$$

where $\mathbf{t}$ is the surface traction vector and $\rho_{\Gamma}$ is the surface density of electric charge. The coupling is introduced by the constitutive equations, assuming linear behavior,

$$
\mathbf{T}=\mathbf{c}^{E}: \mathbf{S}-\mathbf{e}^{t} \cdot \mathbf{E}, \quad \mathbf{D}=\mathbf{e} \cdot \mathbf{S}+\boldsymbol{\beta}^{T} \cdot \mathbf{E}
$$

The elastic $c_{i j}$, dielectric $\beta_{i j}^{T}$ (measured at constant stress) and coupling $g_{i j}$ constants are summarized in Table 1.

The resulting $2-\mathrm{D}$, plain strain model is solved using a FEM formulation based in another one developed by the authors in Pérez-Aparicio et al. 


$\begin{array}{llccccc} & & c_{11} & c_{12} & c_{13} & c_{33} & c_{44} \\ \text { Elastic constants } & {\left[\mathrm{TPa}^{-1}\right]} & 10.99 & -5.36 & -2.22 & 8.24 & 20.16 \\ & & g_{31} & g_{33} & g_{15} & & \\ \text { Coupling constants } & {[\mathrm{MF} / \mathrm{m}]} & -10.691 & 25.111 & 37.981 & & \\ & & \beta_{11}^{T} & \beta_{33}^{T} & & & \\ \text { Dielectric constants } & {[\mathrm{mC}]} & 76.6 & 86.9 & & & \\ \text { Density } & & \rho & & & & \end{array}$

Table 1: Material constants for the PZT-4 plate.

(2007), implemented in the research code FEAP, see Taylor (2003). The main characteristic of this method is the discretization of the basic unknowns,

$$
\begin{aligned}
& \mathbf{u}=\mathbf{N} \mathbf{a}_{\mathbf{u}} \rightarrow \mathbf{S}=\mathbf{B}^{S} \mathbf{a}_{\mathbf{u}} \\
& \phi=\mathbf{N} \mathbf{a}_{\phi} \rightarrow \quad \mathbf{E}=\mathbf{B}^{E} \mathbf{a}_{\phi}
\end{aligned}
$$

where $\mathbf{N}$ are isoparametric shape functions, $\mathbf{a}_{\mathbf{u}}$ and $\mathbf{a}_{\phi}$ the nodal values of the basic unknowns, $\mathbf{B}^{S}=\nabla^{S} \mathbf{N}$ the symmetric part of the mechanical compatibility matrix and $\mathbf{B}^{E}=\nabla \mathbf{N}$ the electric compatibility matrix. Since the excitation used in this paper is dynamic, mechanical mass terms are added to the matrix equations,

$$
\left[\begin{array}{cc}
\mathbf{M} & 0 \\
0 & 0
\end{array}\right]\left\{\begin{array}{l}
\ddot{\mathbf{u}} \\
\ddot{\phi}
\end{array}\right\}+\left[\begin{array}{ll}
\mathbf{K}_{\mathbf{u u}} & \mathbf{K}_{\mathbf{u} \phi} \\
\mathbf{K}_{\phi \mathbf{u}} & \mathbf{K}_{\phi \phi}
\end{array}\right]\left\{\begin{array}{l}
\mathbf{u} \\
\phi
\end{array}\right\}+\left\{\begin{array}{c}
\mathbf{f}_{\mathbf{u}} \\
\mathbf{f}_{\phi}
\end{array}\right\}=\left\{\begin{array}{l}
\mathbf{0} \\
\mathbf{0}
\end{array}\right\}
$$

where the stiffness matrices are defined as,

$$
\begin{array}{rlrl}
\mathbf{K}_{\mathbf{u u}} & =\int_{\Omega} \mathbf{B}^{S} \mathbf{c}^{E} \mathbf{B}^{S} \mathrm{~d} \Omega, & \mathbf{K}_{\mathbf{u} \phi}=\int_{\Omega} \mathbf{B}^{S} \mathbf{e}^{t} \mathbf{B}^{E} \mathrm{~d} \Omega \\
\mathbf{K}_{\phi \mathbf{u}}=\int_{\Omega} \mathbf{B}^{E} \mathbf{e B}^{S} \mathrm{~d} \Omega, & \mathbf{K}_{\phi \phi}=-\int_{\Omega} \mathbf{B}^{E} \boldsymbol{\epsilon}^{S} \mathbf{B}^{T} \mathrm{~d} \Omega
\end{array}
$$

and the mass matrix,

$$
\mathbf{M}=\int_{\Omega} \mathbf{B}^{S} \rho \mathbf{B}^{S} \mathrm{~d} \Omega
$$



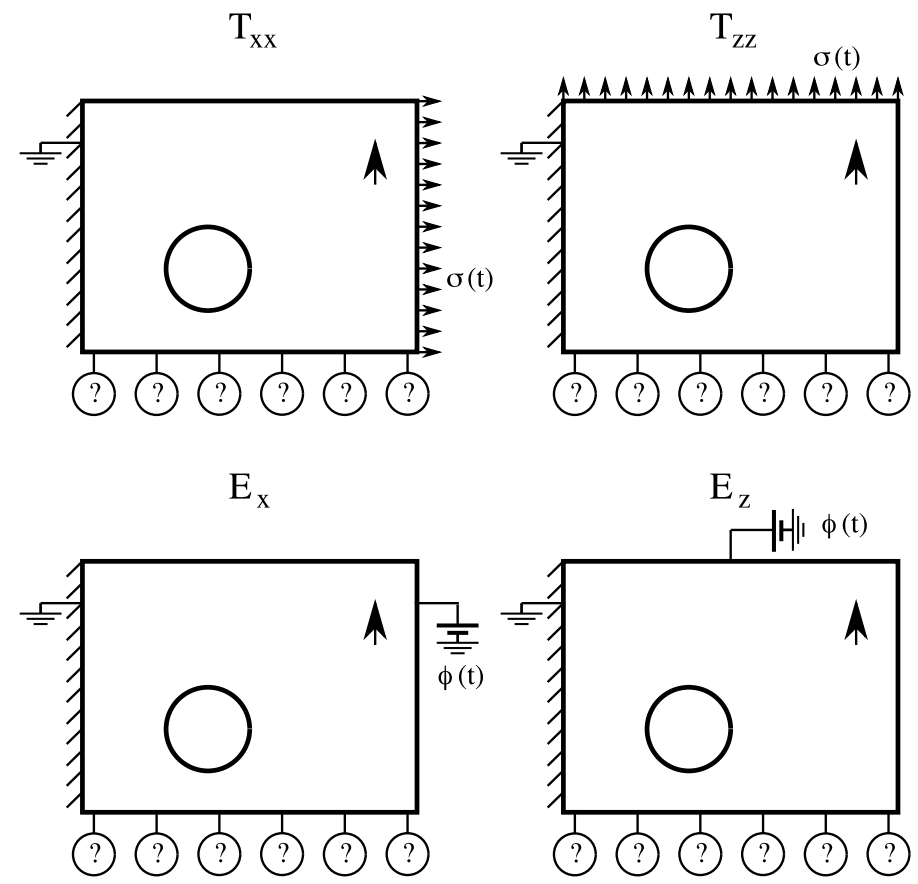

Figure 2: Experimental configurations of excitation-measurement

Two different forces are defined: the mechanical one and the electric field sources,

$$
\mathbf{f}_{\mathbf{u}}=-\int_{\Omega} \mathbf{B}^{S} \mathbf{f} \mathrm{d} \Omega-\int_{\Gamma} \mathbf{B}^{S} \mathbf{t} \mathrm{d} \Gamma, \quad \mathbf{f}_{\phi}=\int_{\Omega} \mathbf{B}^{E} \rho_{\Omega} \mathrm{d} \Omega-\int_{\Gamma} \mathbf{B}^{E} \rho_{\Gamma} \mathrm{d} \Gamma
$$

The transient response is solved by the Newmark- $\beta$ algorithm that requires two user-chosen parameters: $\beta$ to control stability and $\gamma$ for numerical dissipation. According to Zienkiewicz et al. (2005) and for the linearity assumed in this work, this algorithm is unconditionally stable when $\gamma=0.5$ and $\beta=0.25$.

\subsection{Measurement}

The selected excitation/measurement alternative configurations are summarized in Figure 2. The excitation can be mechanical or electrical in two perpendicular directions (four excitation combinations: $T_{x x}, T_{z z}, E_{x}$, $E_{z}$ ), while the measurements along the lower boundary can be mechanical, electrical or combinations of both (seven measurement combinations: 


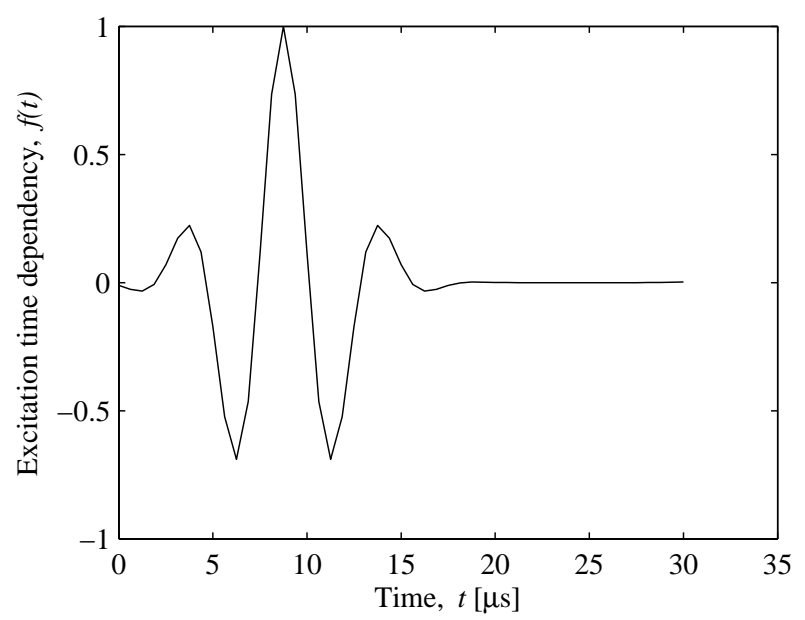

Figure 3: Load time history

$u, v, u|v, u| \phi, v|\phi, u| v \mid \phi$ and $\phi)$.

The excitation is modulated in the time-domain by a pulse defined by a gaussian frequency spectrum, in turn characterized by a central frequency and a bandwidth, which simulates the typical response of piezoelectric actuators. In this work, the central frequency is fixed at $200[\mathrm{kHz}]$ in order to generate several wavelengths within the specimen, whereas the bandwidth is fixed at $50 \%$ of the central frequency,

$$
\sigma(t)=10^{3} f(t)[\mathrm{Pa}], \quad \phi(t)=10^{6} f(t) \quad[\mathrm{V}]
$$

where $f(t)$ is a dimensionless load history constructed from a frequency domain, as shown in Figure 3. The signal time origin is shifted three periods ahead of the maximum amplitude.

The numerically measurement of the response is denoted by $\psi^{N U M}=$ $\left(\mathbf{u}^{N U M}, \phi^{N U M}\right)$, and are obtained solving the forward problem, whereas the experimental one is denoted by $\psi^{E X P}$. No laboratory experiment has been carried out, therefore it is simulated by:

$$
\psi^{E X P}=N\left(\mu_{\psi^{N U M}}, \sigma\right)
$$

where the normally distributed stochastic process $N$ generates random numbers with: a Gaussian distribution with mean $\mu_{\psi^{N U M}}$, obtained through the solving of the forward FEM problem and a standard deviation $\sigma$. The latter is monitorized to study the influence of noise in the final identification. 


\subsection{Cost function}

The Cost Functional ( $\mathrm{CF}$ ), also called objective function, is defined as the $L-2$ norm of the difference between the experimental measurements and those simulated by the numerical procedure, and integrated over the time period interval $T$. An alternative definition of the measurements is proposed, since some measurements have large relative variations along the measured surface. In this definition, the measurements of the same magnitude are normalized by the root-mean square (RMS) of the experimental reading by $\psi_{i}^{\prime E X P}=\psi_{i}^{E X P} / R M S\left(\psi_{i}^{E X P}\right)$ and $\psi_{i}^{\prime N U M}=\psi_{i}^{N U M} / R M S\left(\psi_{i}^{E X P}\right)$,

$$
f=\frac{1}{2 N_{i} T} \int_{T} \sum_{i=1}^{N_{i}}\left(\psi_{i}^{\prime} E X P-\psi_{i}^{\prime N U M}\right)^{2} \mathrm{~d} t
$$

where $N_{i}$ is the number of measuring points (in this work, $N_{i}=25$ ) and $T=30[\mu \mathrm{s}]$, to capture the the development of the main excitation bang plus the return of the fastest mechanical wave.

When genetic or other heuristic search algorithms are used for the minimization, an alternative form of the $\mathrm{CF}$ is defined, improving the algorithm convergence, as argued by Gallego and Rus (2004),

$$
f^{L}=-\lg (f+\varepsilon)
$$

where $\varepsilon$ is a small nondimensional constant (here $\varepsilon=10^{-16}$ ) that ensures the existence of the logarithm when $f$ vanishes.

\subsection{Parametrization}

The location or identification problem can be mathematically formalized as a minimization problem of the $\mathrm{CF}$, then a parametrization of the output is needed. This parametrization consists on defining a set of parameters that sufficiently characterizes the defect, and that become the damage parameters and the result of the IP. The defect is here modeled by a circular cavity with impermeable boundary conditions, see Ou and Chen (2003).

Being this a location and sizing problem, the working parameters are chosen to be the coordinates of the center $x_{0}, z_{0}$ and the radius $r$ of the cavity, grouped in $\mathbf{p}=\left\{x_{0}, z_{0}, r\right\}$, see Figure 4 . The objective final position of the the defect is stored in $\tilde{\mathbf{p}}$.

In order to improve the search algorithm stability, dimensionless alternatives to $\mathbf{p}$ have been defined. They facilitate a linear mapping along a 


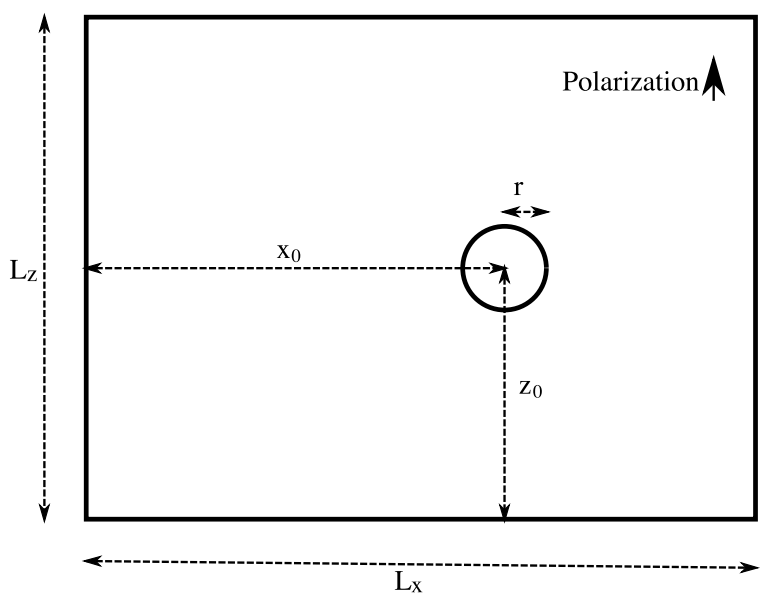

Figure 4: Experimental configuration for the location and sizing of a defect

unitary range of values $\mathbf{p}^{d} \in[0,1]$ to the physical range of allowed values of $\mathbf{p}$, according to the relationship,

$$
\mathbf{p}^{d}=\frac{\mathbf{p}-\mathbf{p}^{\min }}{\mathbf{p}^{\max }-\mathbf{p}^{\min }}
$$

The simulation of experimental measurements corresponds to the case of a cavity with radius $r=20 \%$ of the smallest side, whose center is locate at the non-dimensional coordinates $x_{0}=70 \%, y_{0}=40 \%$.

\subsection{Inverse problem}

The Inverse Problem (IP) can be mathematically understood as a minimization problem (see Figure 1),

$$
\min _{p_{i}^{d}} f\left(\mathbf{p}^{d}\right)
$$

Genetic Algorithm (GA), see Goldberg, D. E. (1989), are employed in this work for the minimization of the CF to obtain the IP output (see Figure 5). In Palma et al. (2009), it was concluded that GA guarantees convergence, whereas gradient-based algorithms strongly depend on the initial guess to be provided. After the redefinition of the $\mathrm{CF}$, the IP solution is obtained as,

$$
\max _{p_{i}^{d}} f^{L}\left(\mathbf{p}^{d}\right)
$$




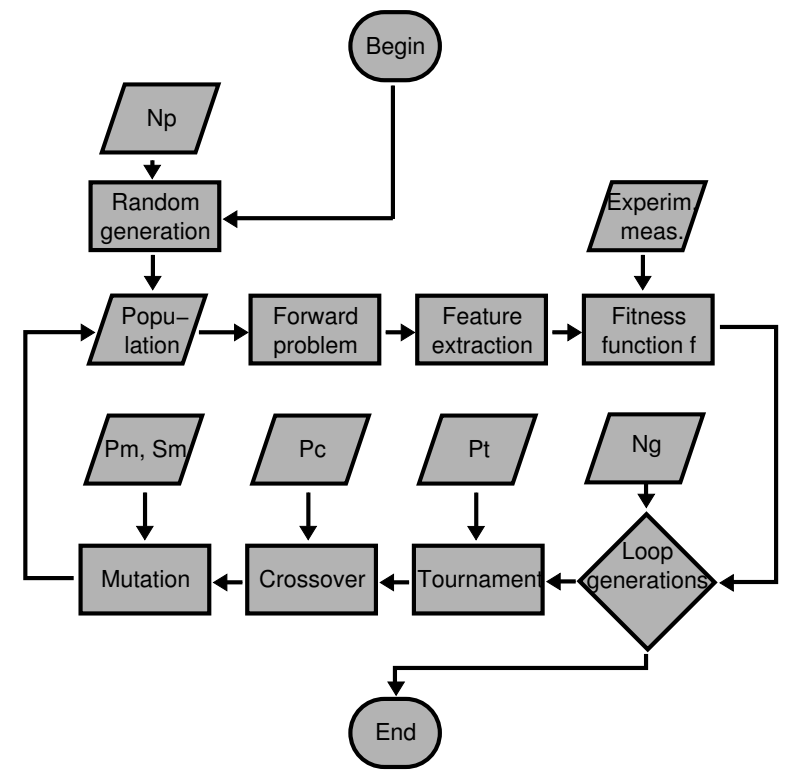

Figure 5: Flow chart of the inverse problem solution by Genetic Algorithms. $N_{p}$ : number of individuals in population; $N_{g}$ : number of generations; $P_{t}$ : probability of tournament; $P_{c}$ : probability of crossover; $P_{m}$ : probability of mutation; $S_{m}$ : scale of mutation.

\subsection{Sensitivity analysis}

The Sensitivity Analysis (SA) permits to understand how model uncertainties and measurement noise affect to the error in the location of the defect. Therefore, SA closes the theory that governs the total error in the IP of damage characterization.

Consider the time-dependent relationship that represents a physical model:

$$
\psi_{i}=\psi_{i}\left(\xi_{j}, p_{k}, t\right)
$$

where, according to the error theory, $\psi_{i}$ are the $i$ dependent or observable variables, $\xi_{j}$ are the $j=1, \ldots, N_{\xi}$ independent or random variables and $p_{k}$ are the $k=1, \ldots, N_{p}$ damage parameters. From the SA theory, it is possible to establish a multilineal relationship as a first approximation (hypothesis validated by Rus et al. (2009)), which corresponds to a Taylor series expansion approximation of several variables,

$$
\psi_{i}(t) \simeq \theta_{i 0}(t)+\sum_{j=1}^{N_{\xi}} \theta_{i j}(t) \xi_{j}+\sum_{k=1}^{N_{p}} \theta_{i k}(t) p_{k}+O\left(\xi_{j}^{2}\right)+O\left(p_{k}^{2}\right)
$$


where $\theta_{i j}$ are the regression coefficients, that approximate the Taylor expansion and can be interpreted as indicators of the relevance of each random variable $\xi_{j}$ with respect $\psi_{i}$,

$$
\theta_{i j}(t) \approx \frac{\partial \psi_{i}(t)}{\partial \xi_{j}}, \quad \theta_{i k}(t) \approx \frac{\partial \psi_{i}(t)}{\partial p_{k}}
$$

These regression coefficients can be calculated by finite differences, as it was reported in Rus et al. (2009).

Assuming that the simulated and experimental measurements can be expressed by (17) and that the random variables are probabilistic magnitudes, the following relationship between experimental and theoretical measurement is obtained,

$$
\psi_{i}^{E X P}(t) \sim \mathcal{N}\left(\mu_{\psi_{i}}(t), \sqrt{\sum_{j=1}^{N_{\xi}} \theta_{i j}^{2}(t) \sigma_{\xi_{j}}^{2}+\sum_{k=1}^{N_{p}} \theta_{i k}^{2}(t) \sigma_{p_{k}}^{2}}\right)
$$

where the multiple linear regression approximation is introduced, together with the error propagation theory.

Equation (19) can be normalized to,

$$
\psi_{i}^{E X P}(t) \sim \psi_{i}^{N U M}(t)+\sqrt{\sum_{j=1}^{N_{\xi}} \theta_{i j}^{2}(t) \sigma_{\xi_{j}}^{2}+\sum_{k=1}^{N_{p}} \theta_{i k}^{2}(t) \sigma_{p_{k}}^{2}} \mathcal{N}(0,1)
$$

if the error of the numerical response is neglected in comparison to the theoretical one $\mu_{\psi}(t)$.

Introducing (20) in (11) and the following weighted squared sum of normally distributed processes is recalled to follow a $\Xi$-square distribution,

$$
\begin{aligned}
& \int_{T}\left(\sum_{j=1}^{N_{\xi}} \theta_{i j}^{2}(t) \sigma_{\xi_{j}}^{2}+\sum_{k=1}^{N_{p}} \theta_{i k}^{2}(t) \sigma_{p_{k}}^{2}\right) \mathcal{N}(0,1) d t \\
\sim & \chi^{2}\left(\sum_{j=1}^{N_{\xi}} \int_{T} \theta_{i j}^{2}(t) \sigma_{\xi_{j}}^{2} d t+\sum_{k=1}^{N_{p}} \int_{T} \theta_{i k}^{2}(t) \sigma_{p_{k}}^{2} d t\right)
\end{aligned}
$$

the expected value of the cost function $f$ is given by,

$$
\mu_{f}=\frac{1}{2 N_{i} T}\left(\sum_{j=1}^{N_{\xi}} \lambda_{j}+\sum_{k=1}^{N_{p}} \lambda_{k}\right)
$$


where $\lambda_{j}$ and $\lambda_{k}$ are scalar sensitivity factors that depend on the regression coefficients and on the standard deviation of the variable $i$,

$$
\lambda_{j}=\sum_{i=1}^{N_{i}} \int_{T} \theta_{i j}^{2}(t) \sigma_{\xi_{j}}^{2} d t, \quad \lambda_{k}=\sum_{i=1}^{N_{i}} \int_{T} \theta_{i k}^{2}(t) \sigma_{p_{k}}^{2} d t
$$

\subsection{Impossible configurations}

In order to avoid impossible configurations, the position of the center of the defect has been defined by nondimensional values, fitting a range defined by a margin equal to the defect semi-length plus $5 \%$ of a representative specimen dimension. This prevents the mesh around the cavity from crossing the solid domain and also avoids quasi-singularities and mesh distortions due to proximity of the cavity to the boundary.

\section{Results}

\subsection{Simulated measurements}

In order to validate the numerical results, two studies on convergence are developed. Figure 6 shows the FEM convergence when the mesh is refined (top) and when the sampling rate is reduced (bottom). These studies permit to choose a compromise between admissible numerical errors (due to mesh size and sampling rate) on one side, versus computational cost on the other.

Figure 7 shows the simulated measurements by FEM for cases without damage and with two levels of damage (defect-sizes $r$ ), keeping the position equal to the real one $\left(x_{0}=70 \%\right.$ and $\left.y_{0}=40 \%\right)$. The combination $E_{z}$ versus $\phi$ is not plotted because the setup is meaningless. It is observed that measurements from the $T_{x x}$ excitation are a priori the least sensitive to damage.

\subsection{Inverse problem}

In this section, the $\mathrm{CF}$ is represented as a starting point in the study to find the optimal excitation/measurements configurations. Figures 8 to 10 show slices of a $4-\mathrm{D}$ space. Note that the defect is represented by three parameters (damage parameters), the $\mathrm{CF}$ is a warped surface not easy to visualize. Therefore, two parameters are used, leaving the remaining one fixed at the true value. The $\mathrm{CF}$ is computed simulating measurements with a $10 \%$ gaussian error. As it is observed in the figures, the CF exhibits several 

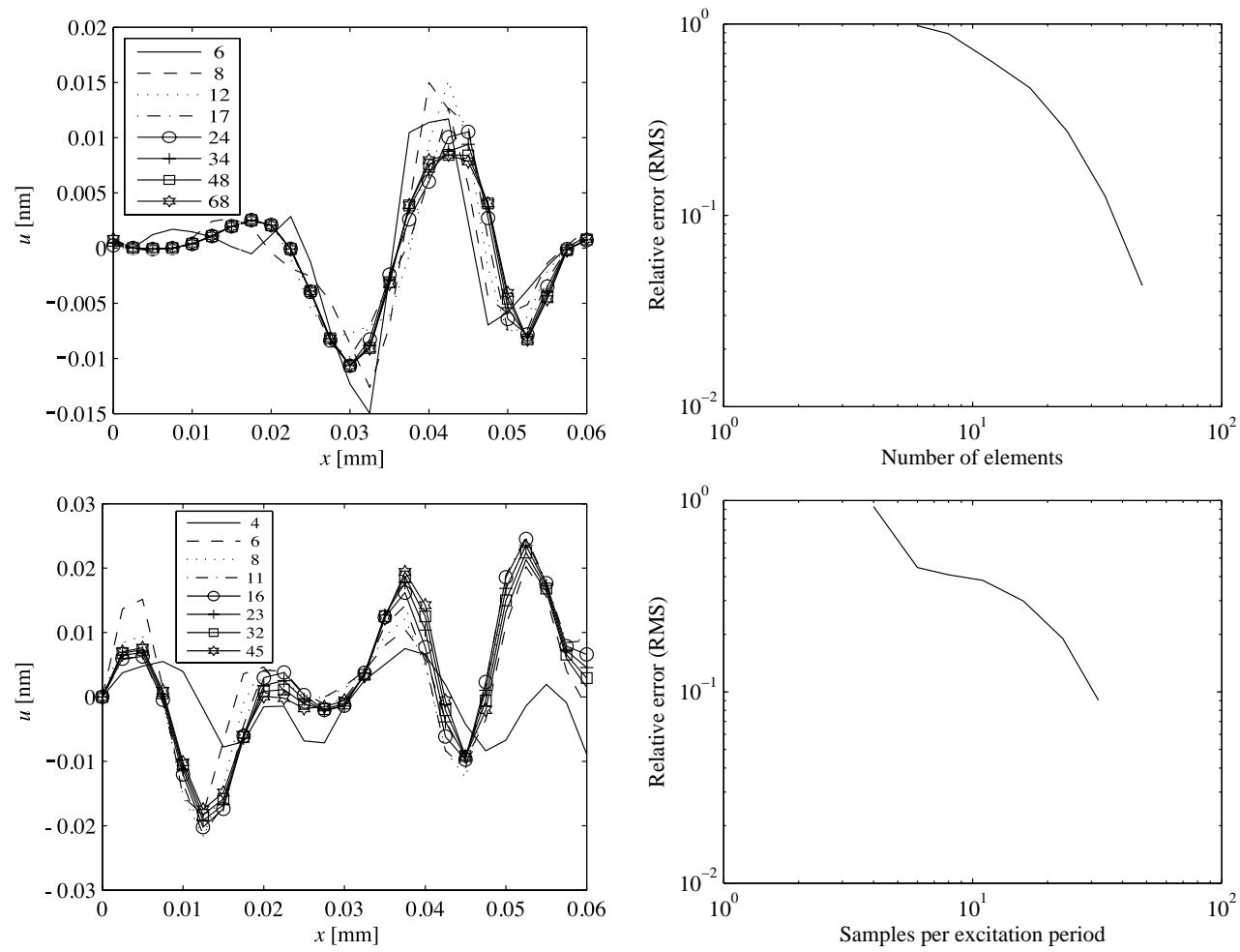

Figure 6: Convergence study for mesh size (top) and sampling rate (bottom) 

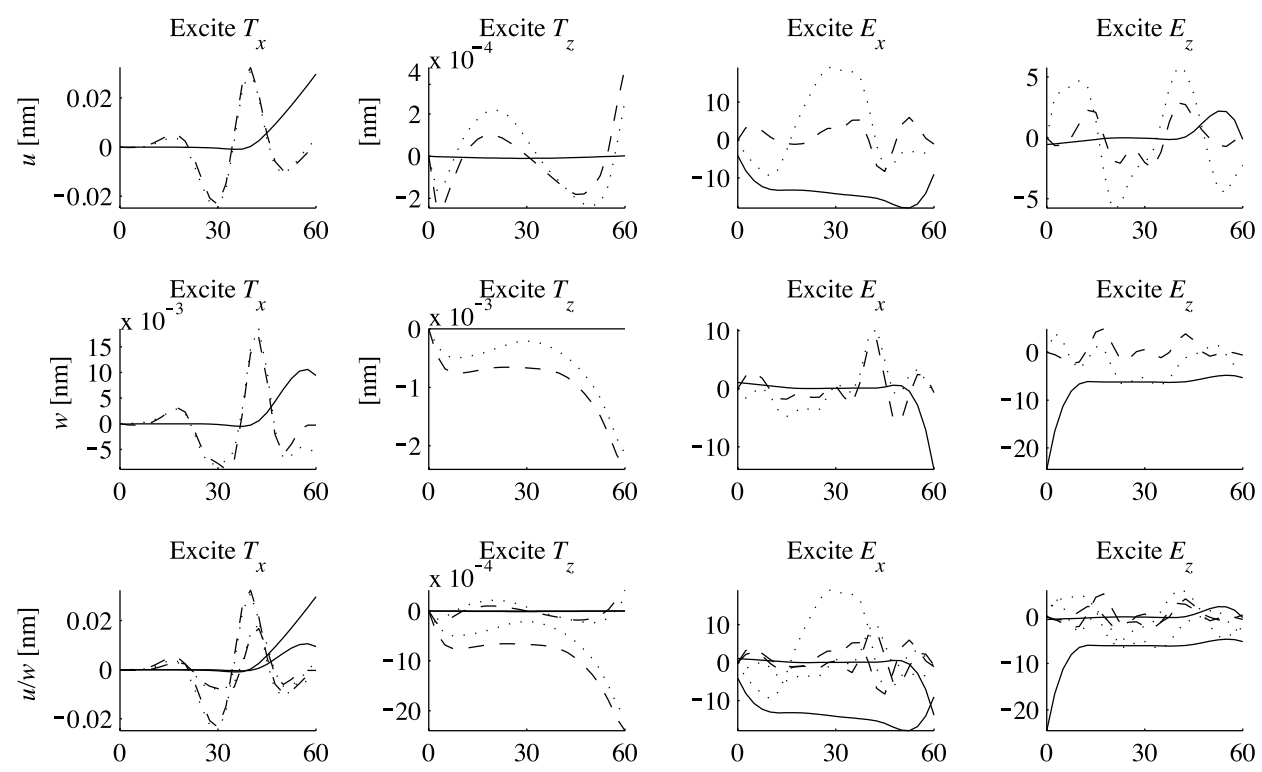

Excite $T_{x}$

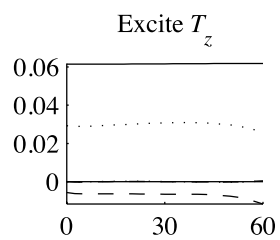

Excite $E_{x}$
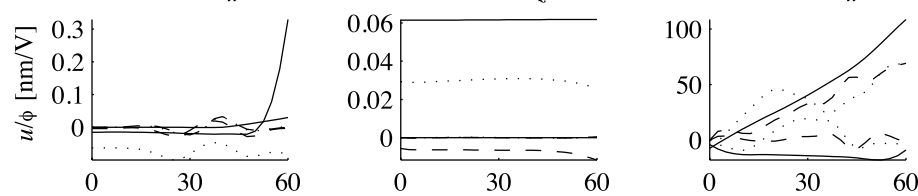

Excite $E$

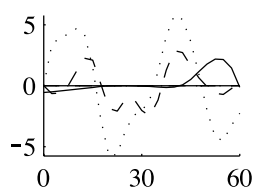

Excite $T_{x}$

Excite $T_{z}$
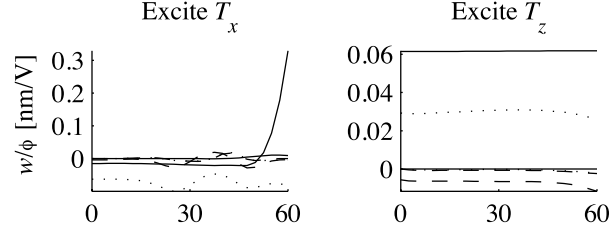

Excite $E_{x}$

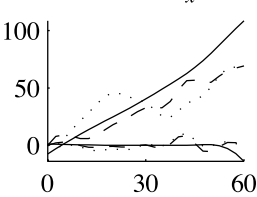

Excite $E$

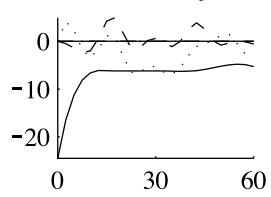

Excite $T_{x}$

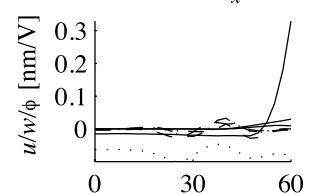

Excite $T_{z}$

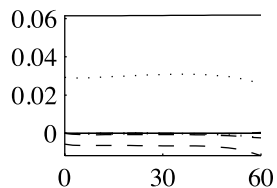

Excite $E_{x}$

Excite $E_{z}$
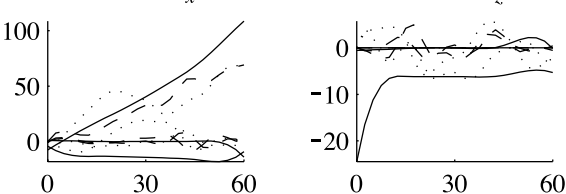

Excite $T_{x}$
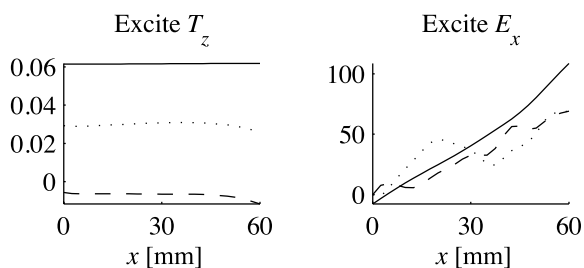

Excite $E$
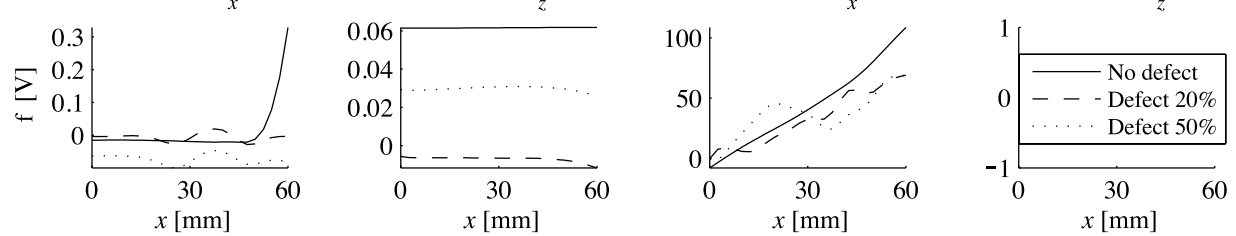

Figure 7: Simulated measurements for all excitation/measurements configurations at $t=$ $15 \mu \mathrm{s}$ 
local minima, especially visible in all $x_{0}-y_{0}$ plots for the $E_{x}$ excitation and measurements containing $\phi$. Furthermore, some damage parameters show a strong dependency, for instance $x_{0}$ to $r$ under $T_{x x}$ excitation. This is due to the fact that combinations of $x_{0}$ and $r$ along the valley yield similar response and low $\mathrm{CF}$ values, and it is difficult to decouple these values (i.e., find the local minima). Finally, very small defects $r \approx 0$ invariably yield low $\mathrm{CF}$ values, indicating a low limit to the detectable defect when noise is present.

\subsection{Search algorithm convergence}

In this section the search algorithm is studied, adjusting the GA parameters to achieve a computational efficiency and to guarantee a good convergence.

Figure 11 shows the evolution of the best individual and the population mean versus each generation. To ensure that the solution does not converge to a local minimum, genetic diversity was injected by the mutation and crossover parameters.

In order to perform the convergence analysis, the distance between the parameters that characterize the real and the calculated defects are defined by the following norm,

$$
d=\frac{\sqrt{\sum_{i=1}^{N}\left(\tilde{p}_{i}-p_{i}^{c}\right)^{2}}}{\sum_{i=1}^{N} \tilde{p}_{i}}
$$

Figure 12 shows the number of generations versus the population size. To guarantee the convergence to a global optimum, while establishing a compromise between IP error and computational cost, the GA selected parameters are shown in Table 2.

\begin{tabular}{lr} 
Parameter & Value \\
\hline \hline Population size & 10 \\
Number of generations & 40 \\
Probability of crossover & 0.80 \\
Probability of mutation & 0.10 \\
Probability of selection & 0.70
\end{tabular}

Table 2: Parameters used for the Genetic Algorithm search 

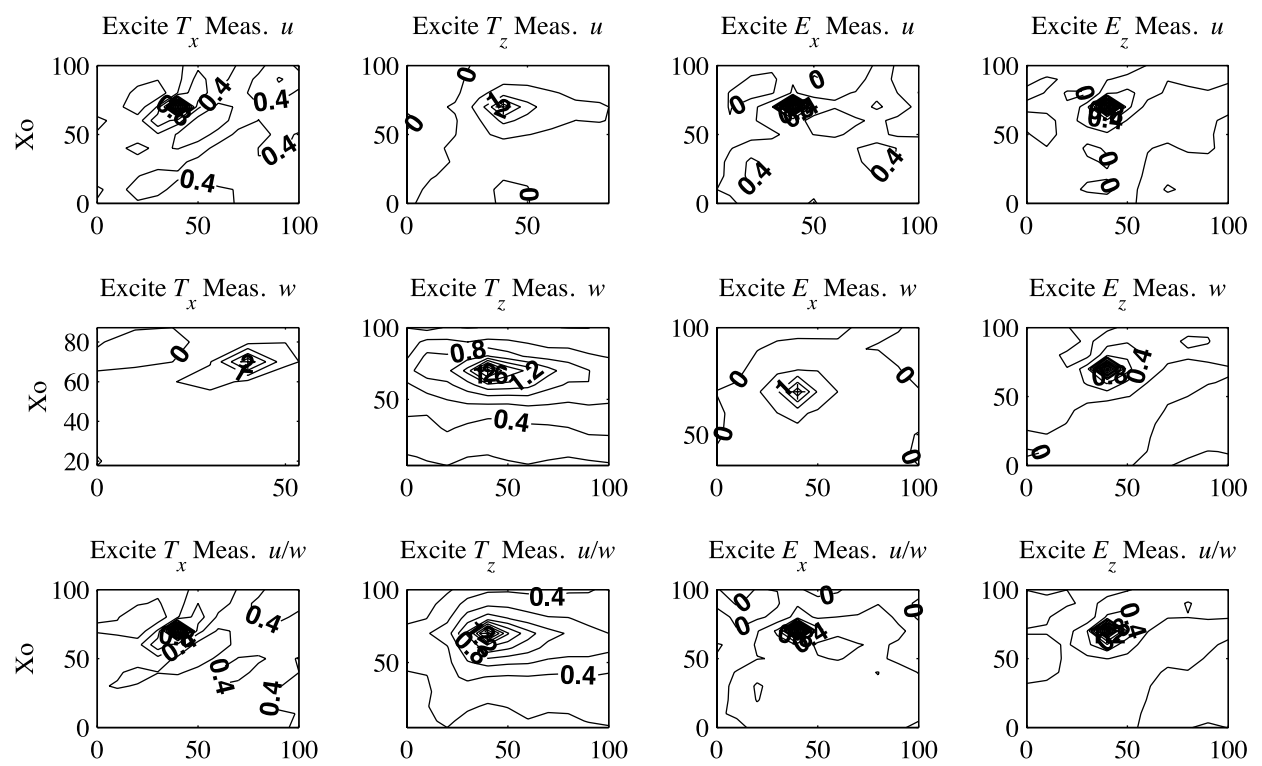

Excite $E_{x}$ Meas. $u / w$

Excite $E$ Meas. $u / w$
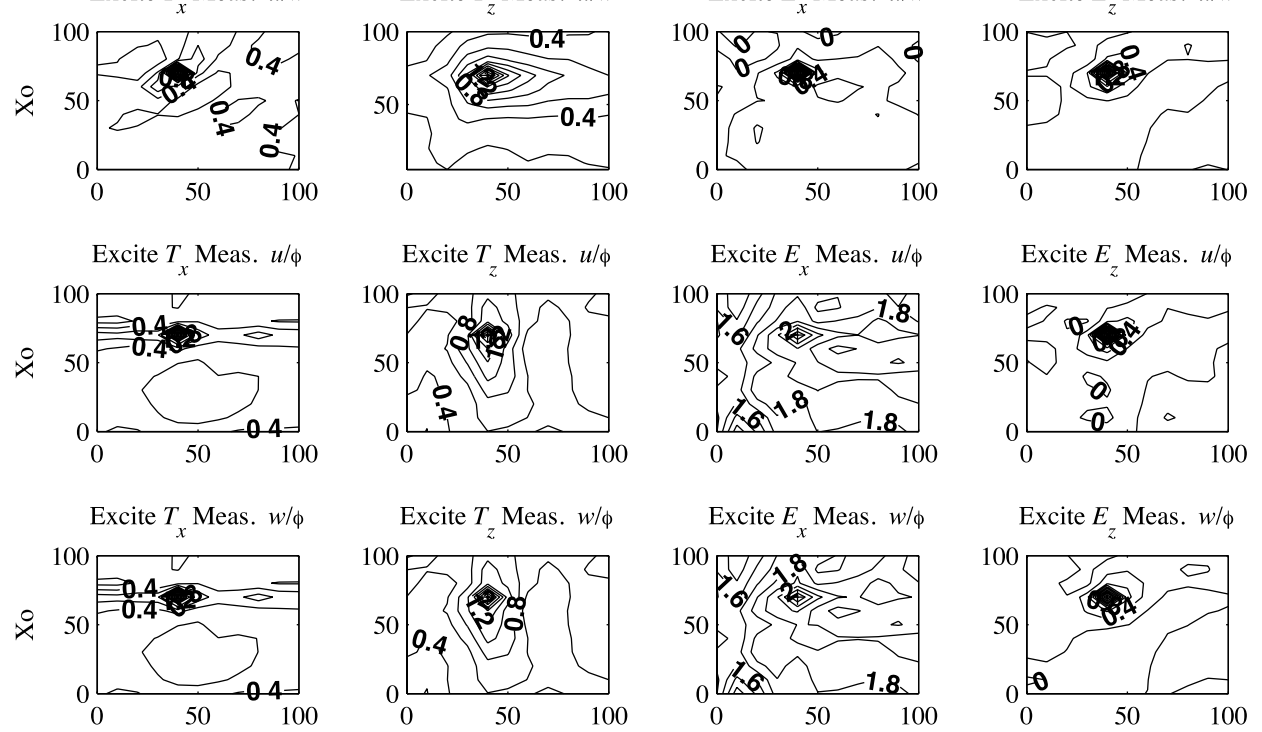

Excite $T_{x}$ Meas. $u / w / \phi$

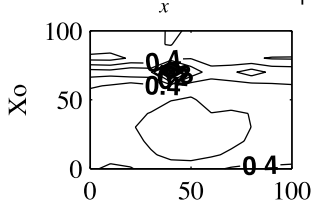

Excite $T_{z}$ Meas. $u / w / \phi$

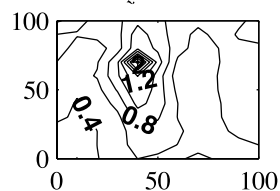

Excite $E_{x}$ Meas. $u / w / \phi$

Excite $E_{z}$ Meas. $u / w / \phi$
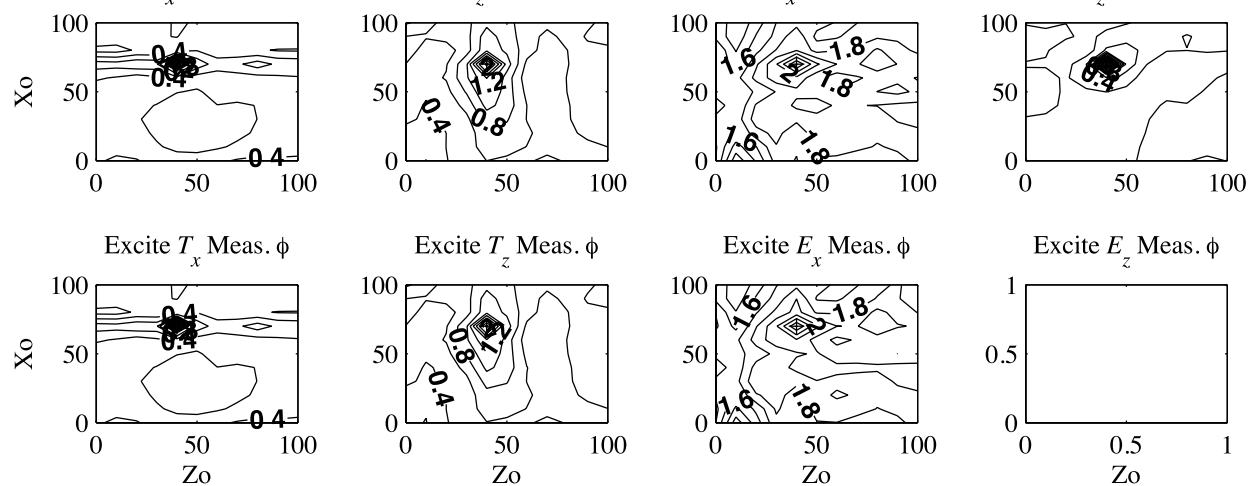

Figure 8: $\mathrm{CF}$ as a function of defect parameters 

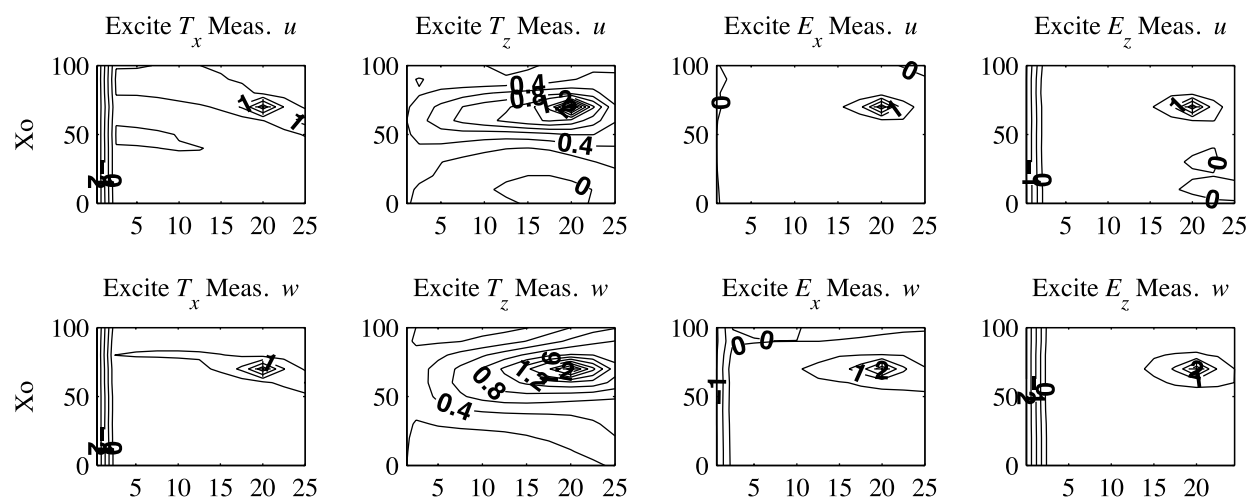

Excite $T_{x}$ Meas. $u / w$
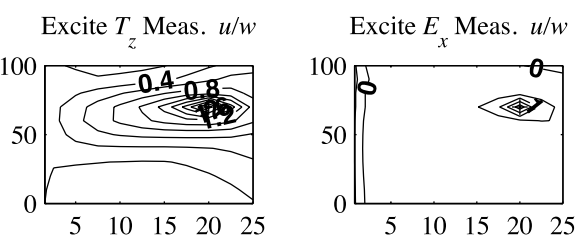

Excite $E_{z}$ Meas. $u / w$

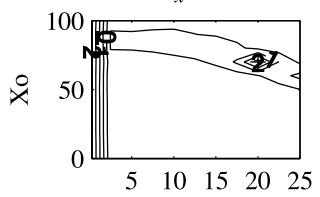

Excite $T_{z}$ Meas. $u / \phi$
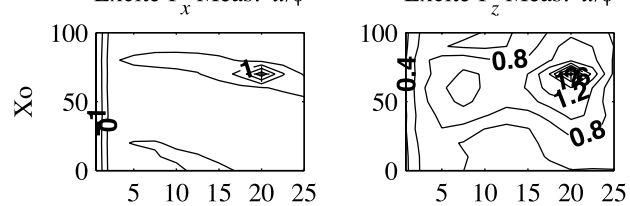

Excite $E_{x}$ Meas. $u / \phi$
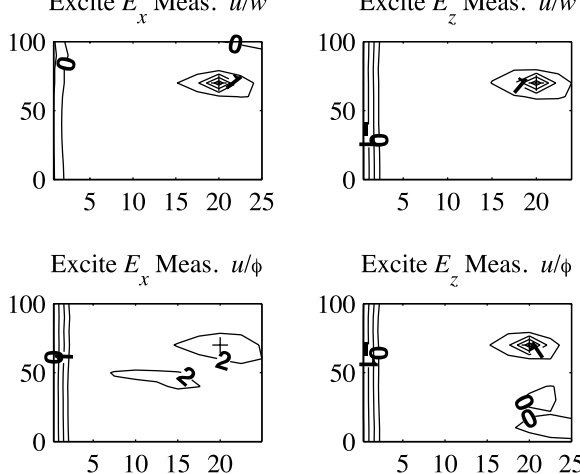

Excite $E$ Meas. $u / \phi$
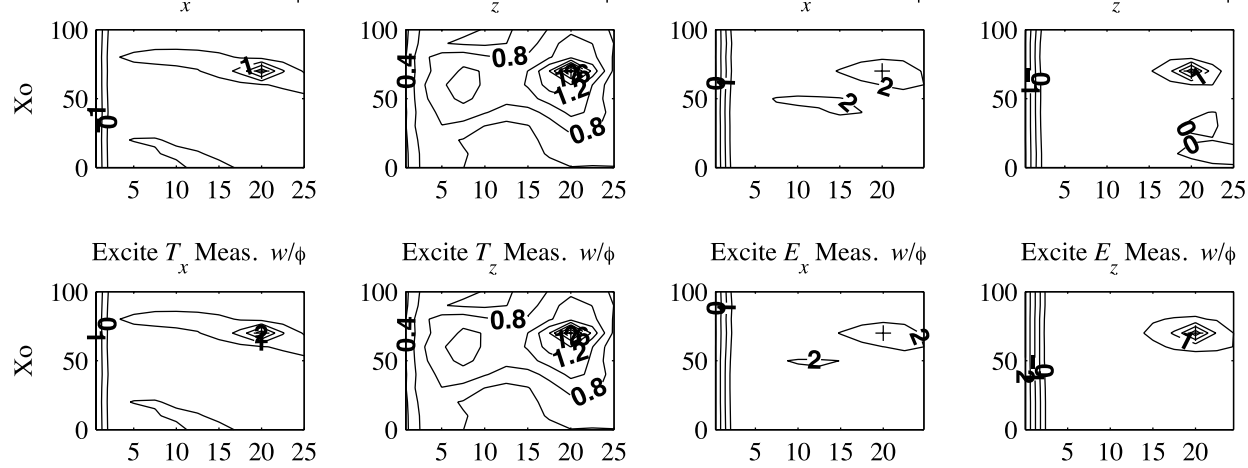

Excite $T_{z}$ Meas. $w / \phi$
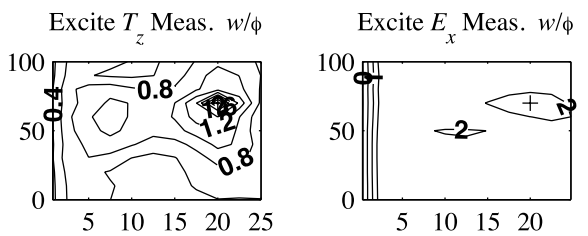

Excite $E$ Meas. $w / \phi$

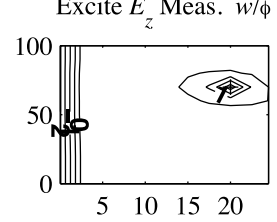

Excite $T_{x}$ Meas. $u / w / \phi$

Excite $T_{z}$ Meas. $u / w / \phi$
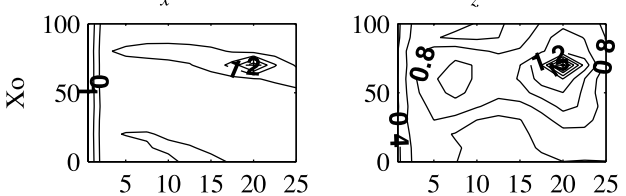

Excite $E_{x}$ Meas. $u / w / \phi$

Excite $E_{z}$ Meas. $u / w / \phi$
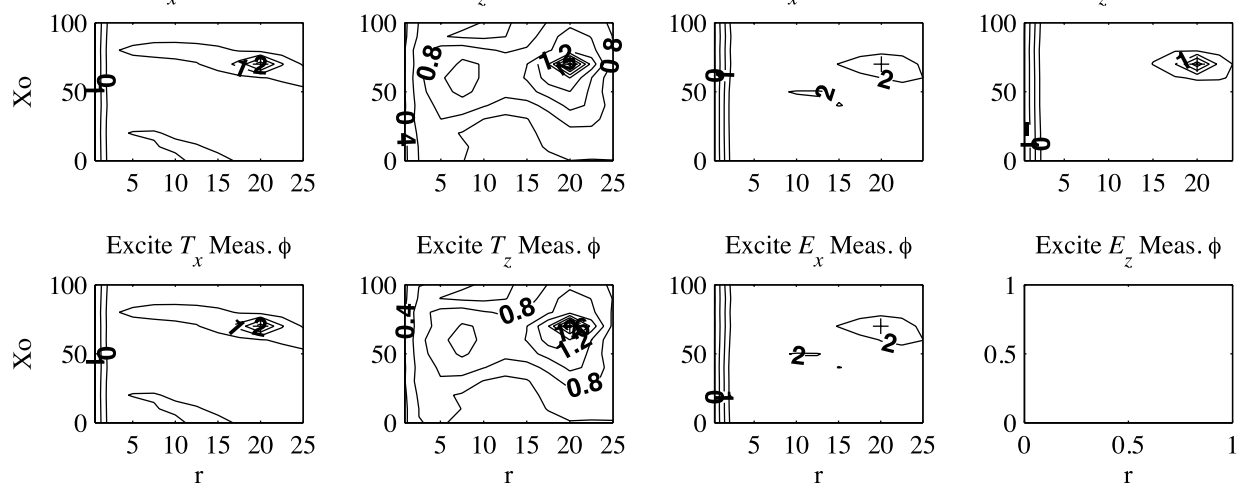

Figure 9: $\mathrm{CF}$ as a function of defect parameters 
Excite $T_{x}$ Meas. $u$

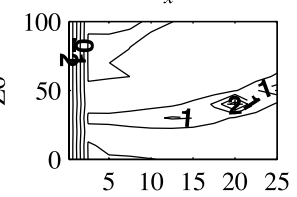

Excite $T_{x}$ Meas. $w$

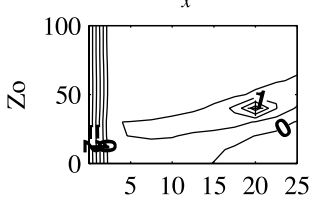

Excite $T_{x}$ Meas. $u / w$

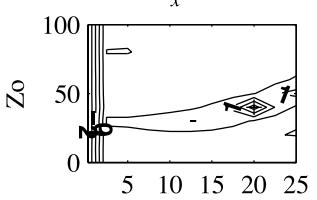

Excite $T_{x}$ Meas. $u / \phi$

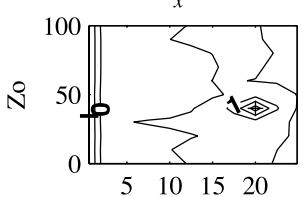

Excite $T_{x}$ Meas. $w / \phi$

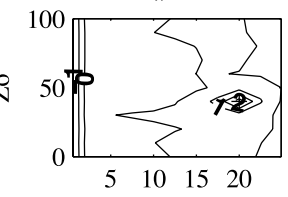

Excite $T_{x}$ Meas. $u / w / \phi$
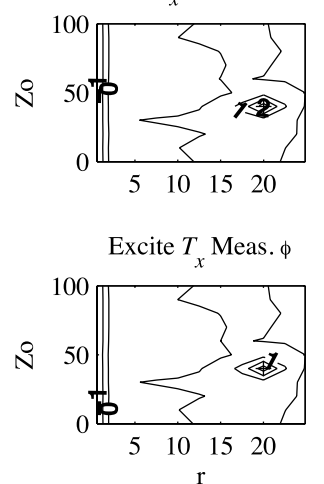

Excite $T$ Meas. $u$

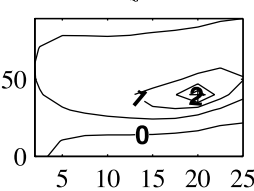

Excite $T_{z}$ Meas. $w$

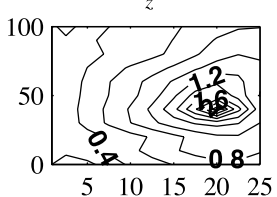

Excite $T$ Meas. $u / w$

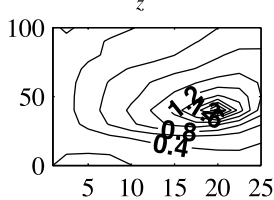

Excite $T$ Meas. $u / \phi$

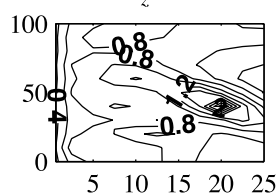

Excite $T_{z}$ Meas. $w / \phi$

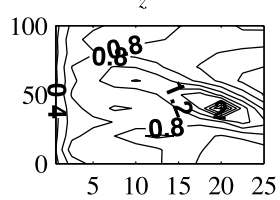

Excite $T_{z}$ Meas. $u / w / \phi$

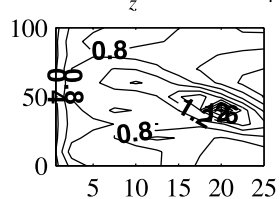

Excite $T$ Meas. $\phi$

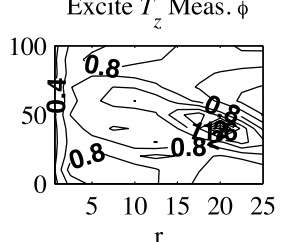

Excite $E_{x}$ Meas. $u$
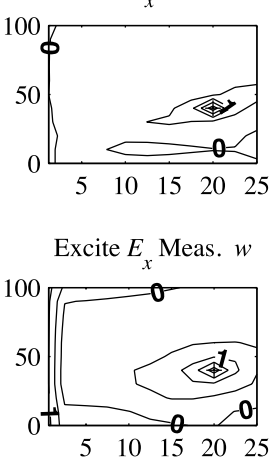

Excite $E_{x}$ Meas. $u / w$

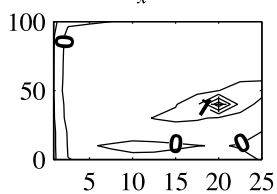

Excite $E_{x}$ Meas. $u / \phi$

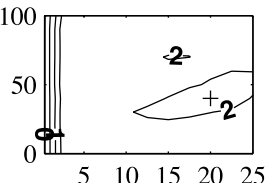

Excite $E_{x}$ Meas. $w / \phi$

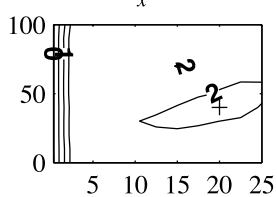

Excite $E_{x}$ Meas. $u / w / \phi$
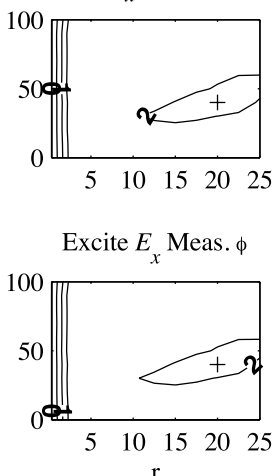

Excite $E$ Meas. $u$

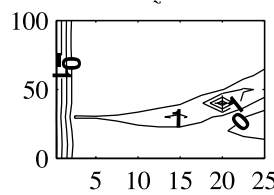

Excite $E$ Meas. $w$

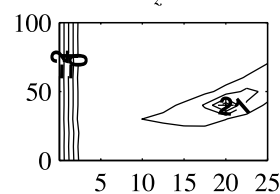

Excite $E_{z}$ Meas. $u / w$

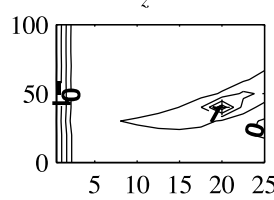

Excite $E_{z}$ Meas. $u / \phi$

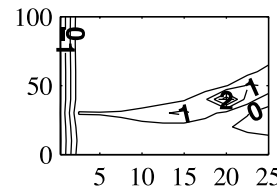

Excite $E_{z}$ Meas. $w / \phi$

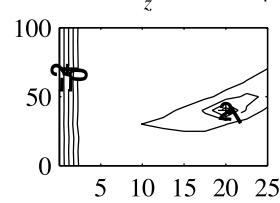

Excite $E_{z}$ Meas. $u / w / \phi$

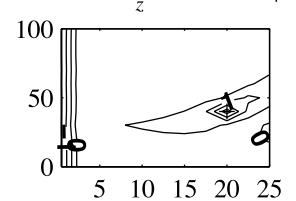

Excite $E$ Meas. $\phi$

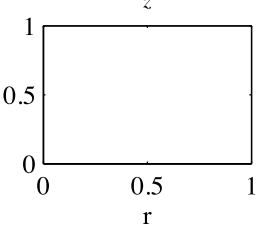

Figure 10: $\mathrm{CF}$ as a function of defect parameters 

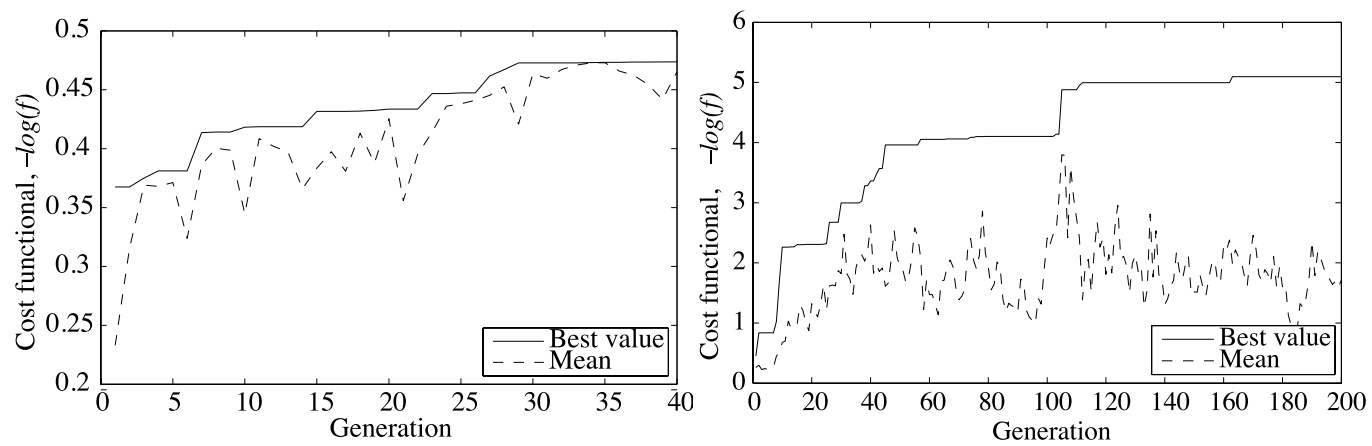

Figure 11: Evolution of the Genetric Algorithm for: 5 individuals, 40 generations (left), and 10 individuals, 200 generations (right)
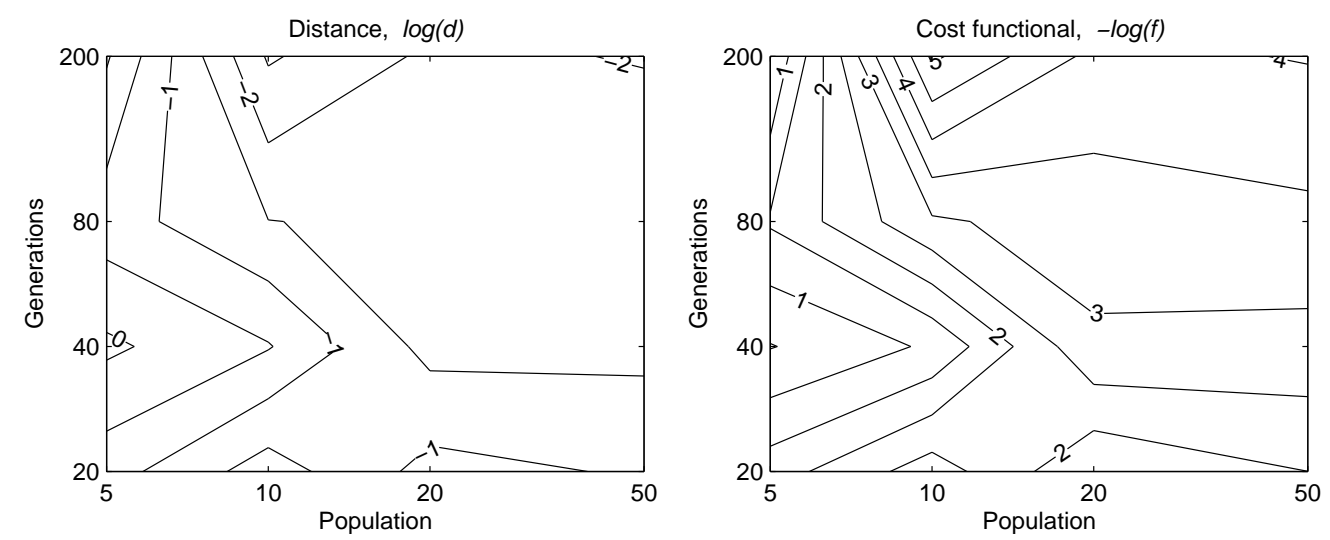

Figure 12: Convergence analysis of Genetic Algorithms for an Inverse Problem. 


\subsection{Sensitivity analysis}

In this section, a SA based on the $\theta_{i j}(t)$ and $\theta_{i k}(t)$ functions from (18) and sensitivity factors $\lambda$ from (23) is performed.

Figure 13 and Figure 14 show the approximation by finite differences of the regression coefficients between measurement values and: i) model parameters (Figure 13) and ii) damage parameters (Figure 14). The variation in shape and value of these curves is an indicator of the measurement sensitivity to each system uncertainty or to each working variable, respectively.

Figures 15 and 16 show the sensitivity factor $\lambda$ for a set of possible excitation/measurement configurations. This value succinctly quantifies which parameters are responsible to the largest extent of the error in the identification. The model parameters related to smaller $\lambda$ values need to be known with high precision, since they will affect the $\mathrm{CF}$ to a larger extent, and can falsify the final solution, if they are too large. On the other hand, the damage parameters related to larger $\lambda$ values are candidates to be known with low precision, since they are clearly dependent on changes in the $\mathrm{CF}$.

Since not all possible defects have the same identifiability, i.e. different defect locations and sizes may generate variable sensitivity factors $\lambda$, a statistical interpretation of the sensitivity has been developed using a Monte Carlo algorithm (MC). Assuming that the defect characterization parameters are uniformly valued stochastic variables (non deterministic), the sensitivity factor $\lambda$ is also an stochastic variable. The probability density function of $\lambda$ can be calculated using $\mathrm{MC}$, but this algorithm involves many finite differences and FEM computations. Therefore, a Latin Hypercube sampling is adopted to reduce the size of the computation to 100 samples (which reduces the error in the mean estimate to $1 / \sqrt{100}=10 \%$ of the standard deviation). Furthermore, the Latin Hypercube sampling is optimized by the correlation criterion with 1000 iterations. The percentile 5 of the probabilistic distributions is estimated as a pessimistic estimate of the sensitivity.

The MC results are shown in Figures 17 and 18. These figures are in agreement with the deterministic ones for the selected experimental configurations.

To numerically compare the former sensitivities, Table 3 summarizes the estimator $\Lambda$ between the sum of model sensitivities $\lambda_{j}$ multiplied by the combined inverse damage sensitivity $\lambda_{k}$. The last term is included since higher damage sensitivities correspond to better and higher overall sensitivity. 

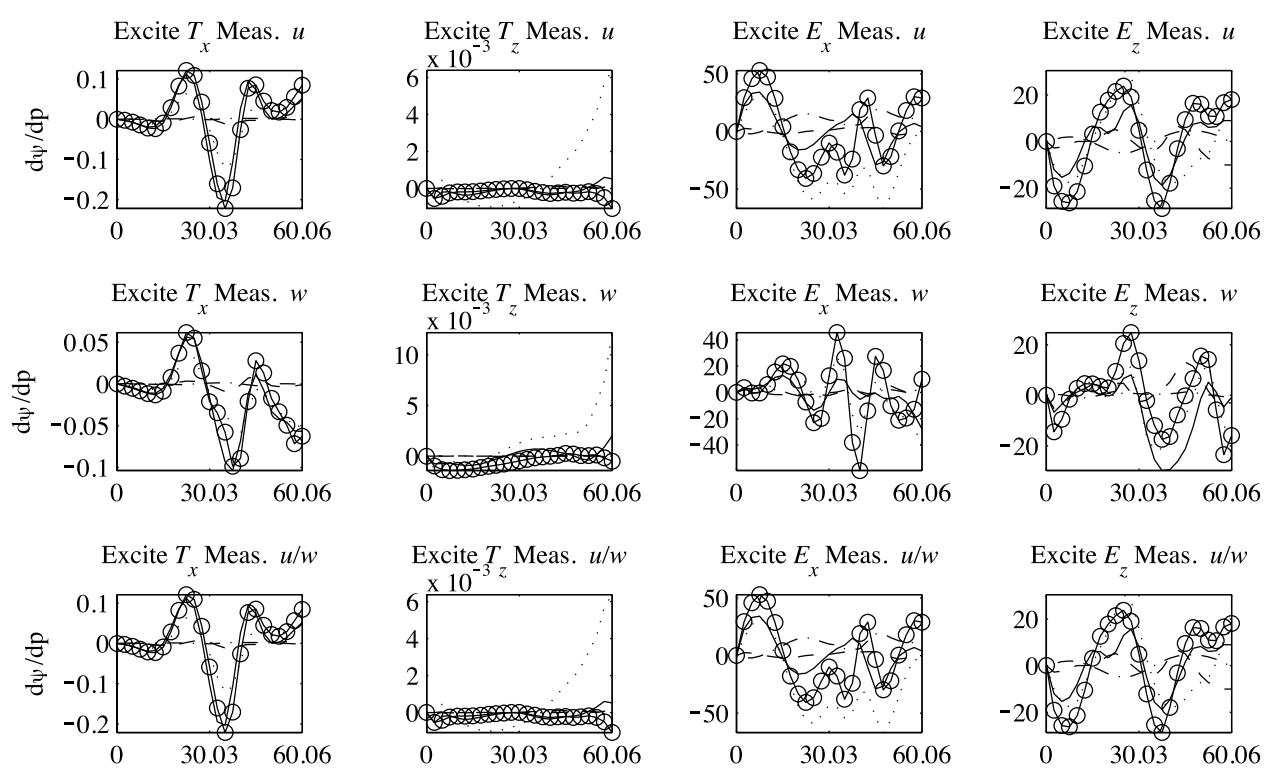

Excite $E_{x}$ Meas. $u / w$ Excite $E_{z}$ Meas. $u / w$
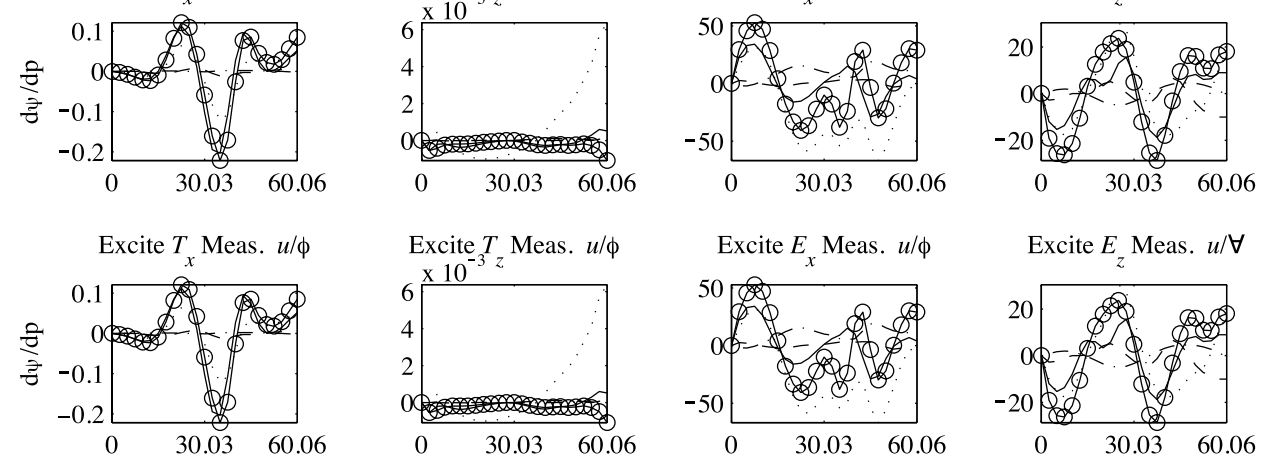

Excite $E_{z}$ Meas. $u / \forall$
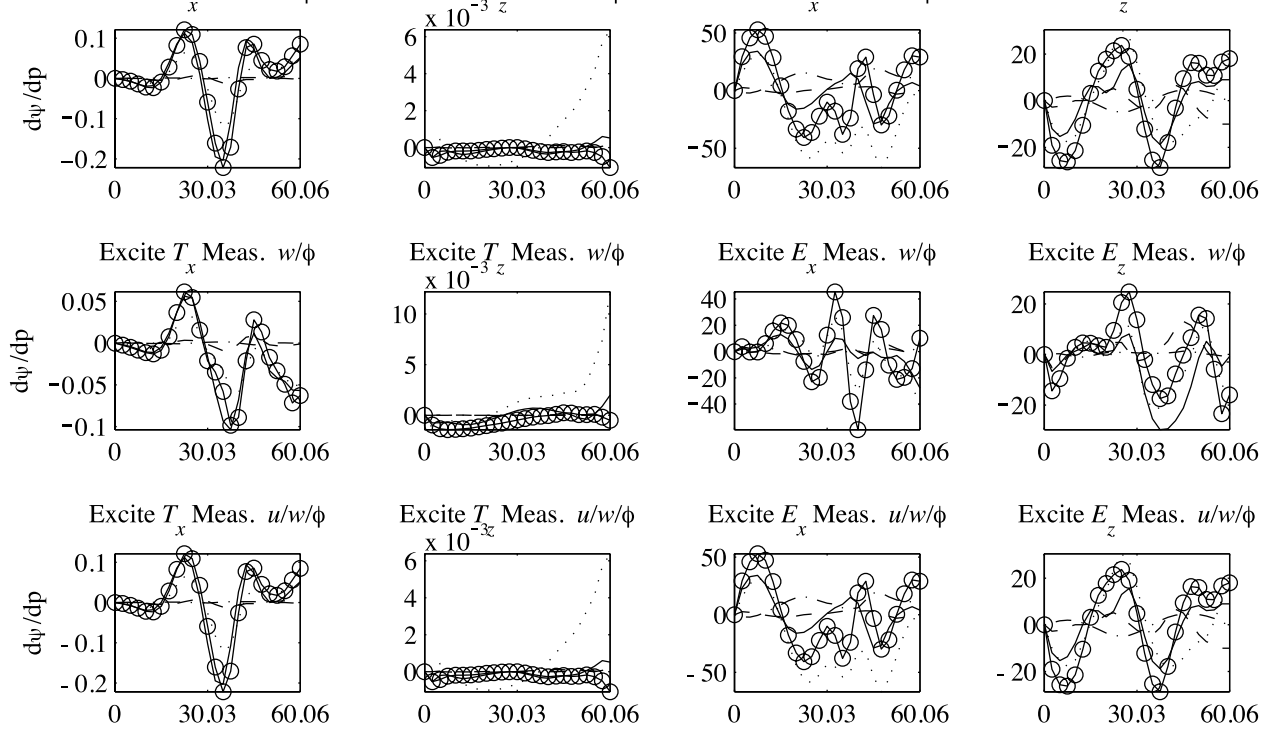

Excite $E_{x}$ Meas. $u / w / \phi$
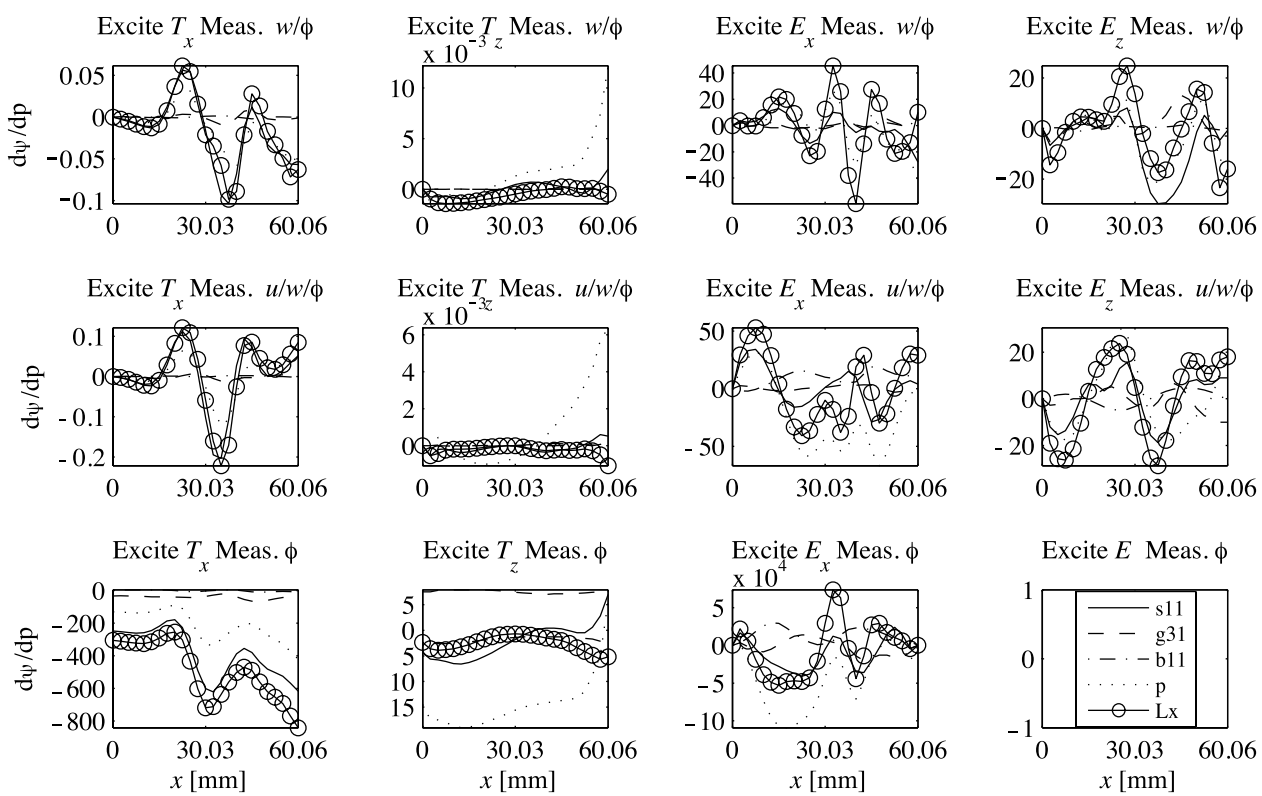

Figure 13: Measurement sensitivity to mechanical/geometrical model parameters 

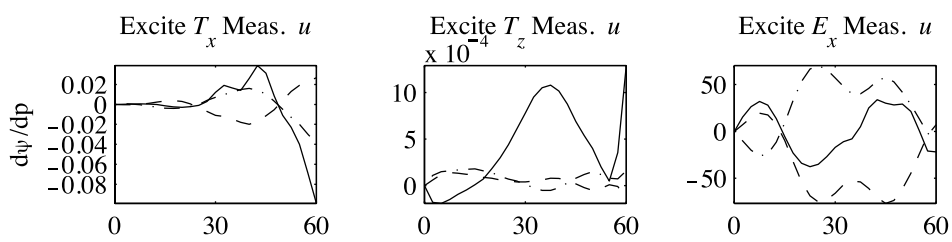

Excite $E_{z}$ Meas. $u$

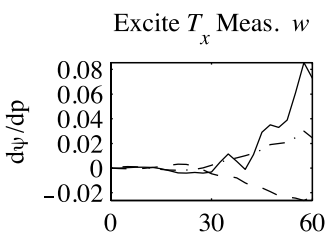

Excite $T_{z}$ Meas. $w$
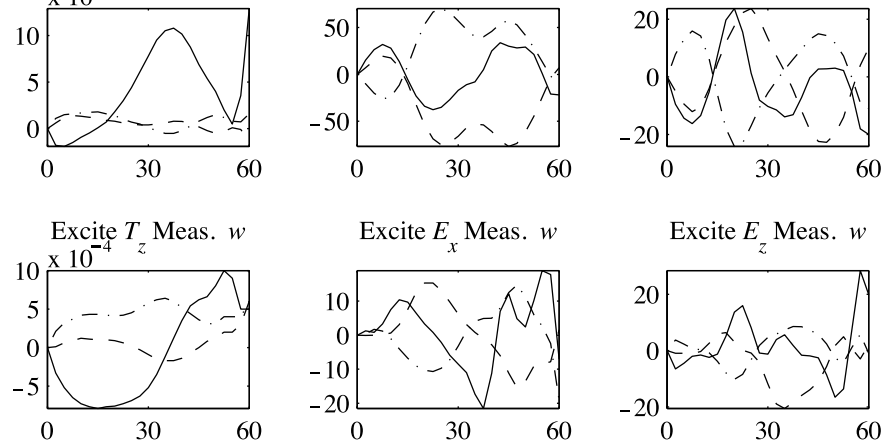

Excite $T_{x}$ Meas. $u / w$

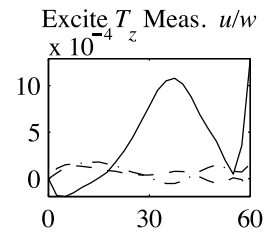

Excite $E_{x}$ Meas. $u / w$
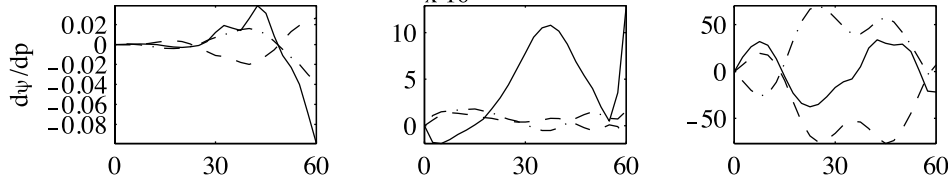

Excite $E$ Meas. $w$

Excite $T_{x}$ Meas. $u / \phi$

Excite $T_{z}$ Meas. $u / \phi$

Excite $E_{x}$ Meas. $u / \phi$

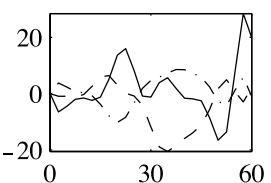

Excite $E_{z}$ Meas. $u / w$

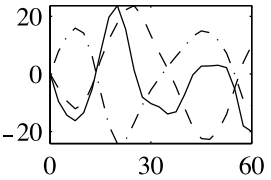

Excite $E_{z}$ Meas. $u / \phi$
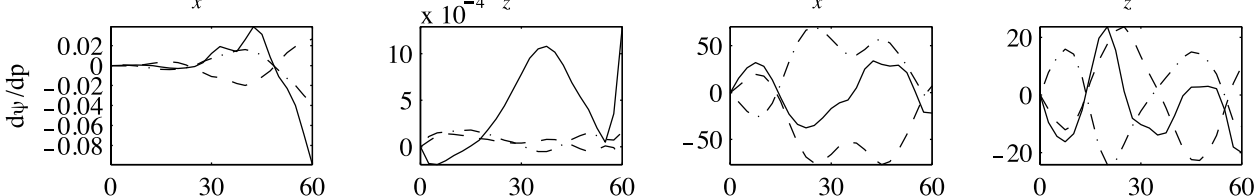

Excite $T_{x}$ Meas. $w / \phi$
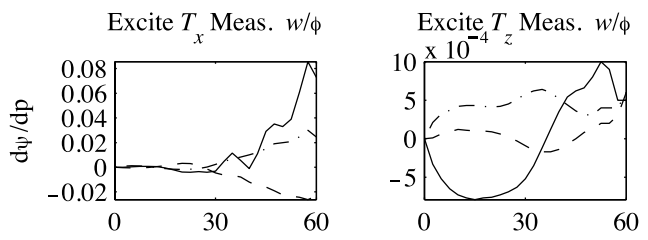

Excite $E_{x}$ Meas. $w / \phi$

Excite $E_{z}$ Meas. $w / \phi$
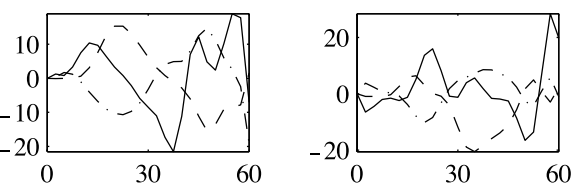

Excite $T_{x}$ Meas. $u / w / \phi$

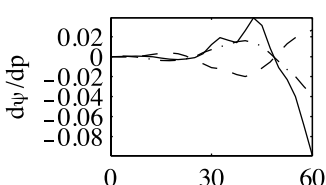

Excite $T$ Meas. $u / w / \phi$

Excite $E_{x}$ Meas. $u / w / \phi$

Excite $E_{z}$ Meas. $u / w / \phi$
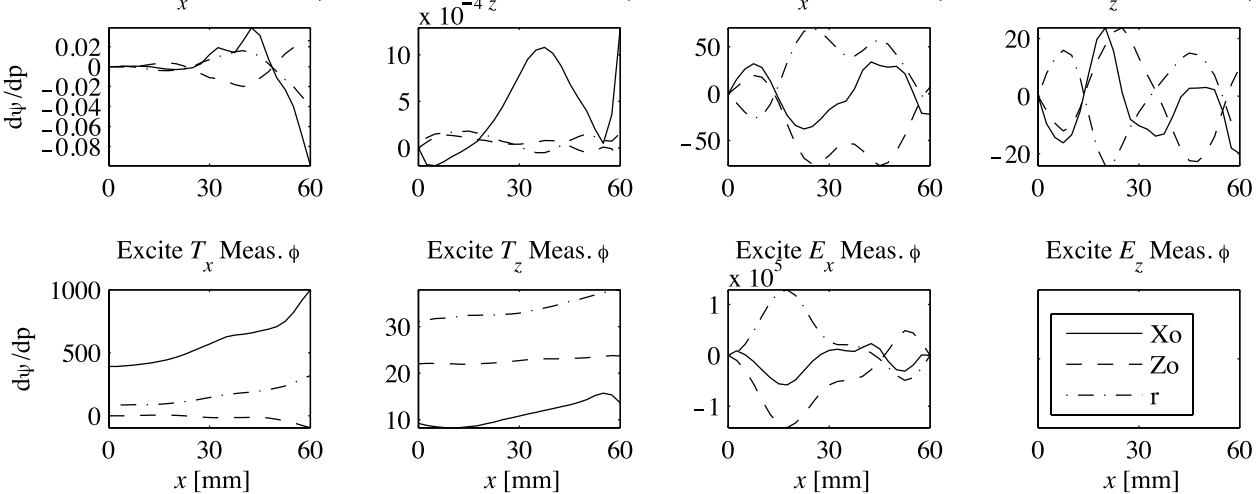

Excite $E_{z}$ Meas. $\phi$

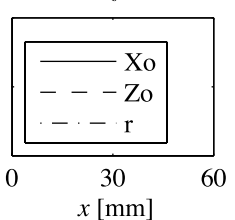

Figure 14: Measurement sensitivity to damage parameters 

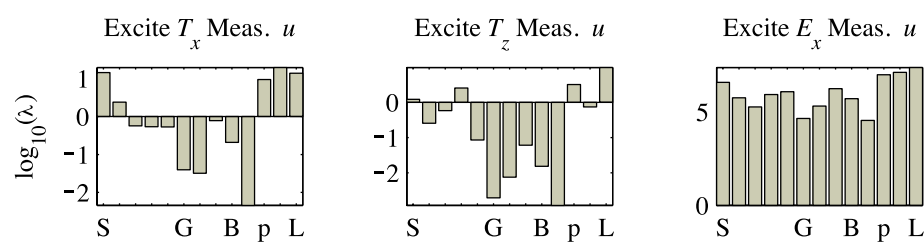

Excite $E_{z}$ Meas. $u$

Excite $T_{x}$ Meas. $w$

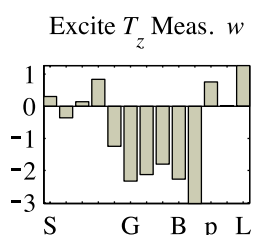

Excite $E_{x}$ Meas. $w$
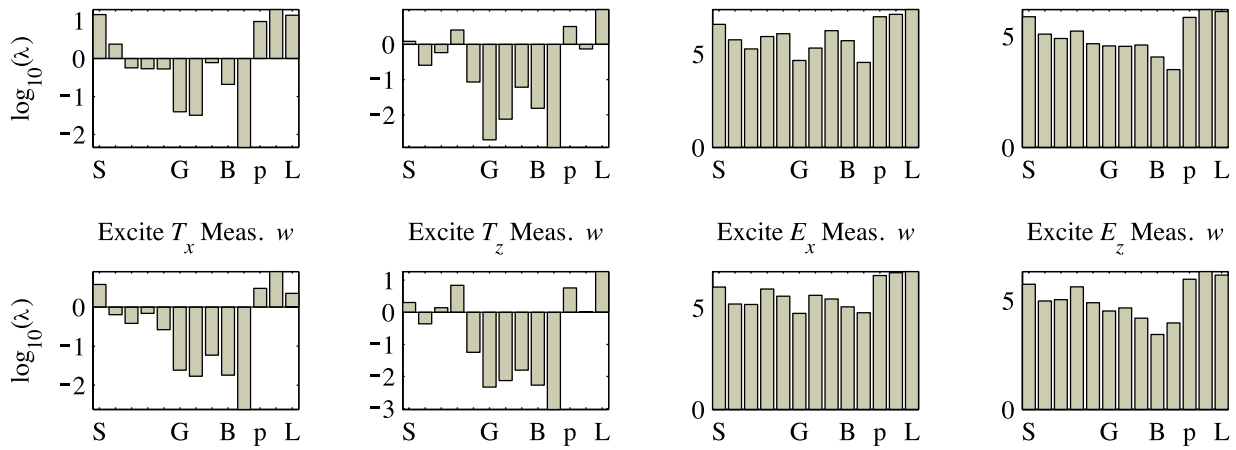

Excite $E_{z}$ Meas. $w$

Excite $T_{x}$ Meas. $u / w$

Excite $T_{z}$ Meas. $u / w$

Excite $E_{x}$ Meas. $u / w$
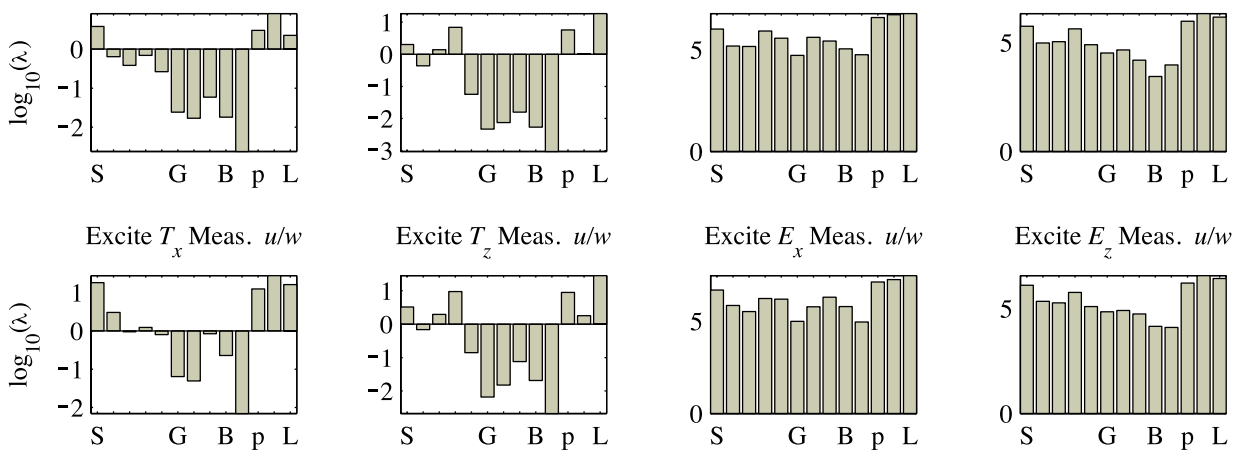

Excite $T_{x}$ Meas. $u / \phi$

Excite $T_{z}$ Meas. $u / \phi$

Excite $E_{x}$ Meas. $u / \phi$
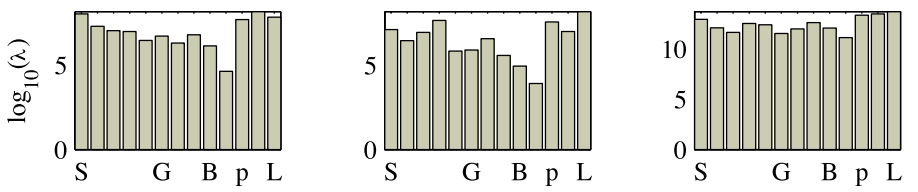

Excite $E_{z}$ Meas. $u / w$

Excite $T_{x}$ Meas. $w / \phi$

Excite $T_{z}$ Meas. $w / \phi$

Excite $E_{x}$ Meas. $w / \phi$
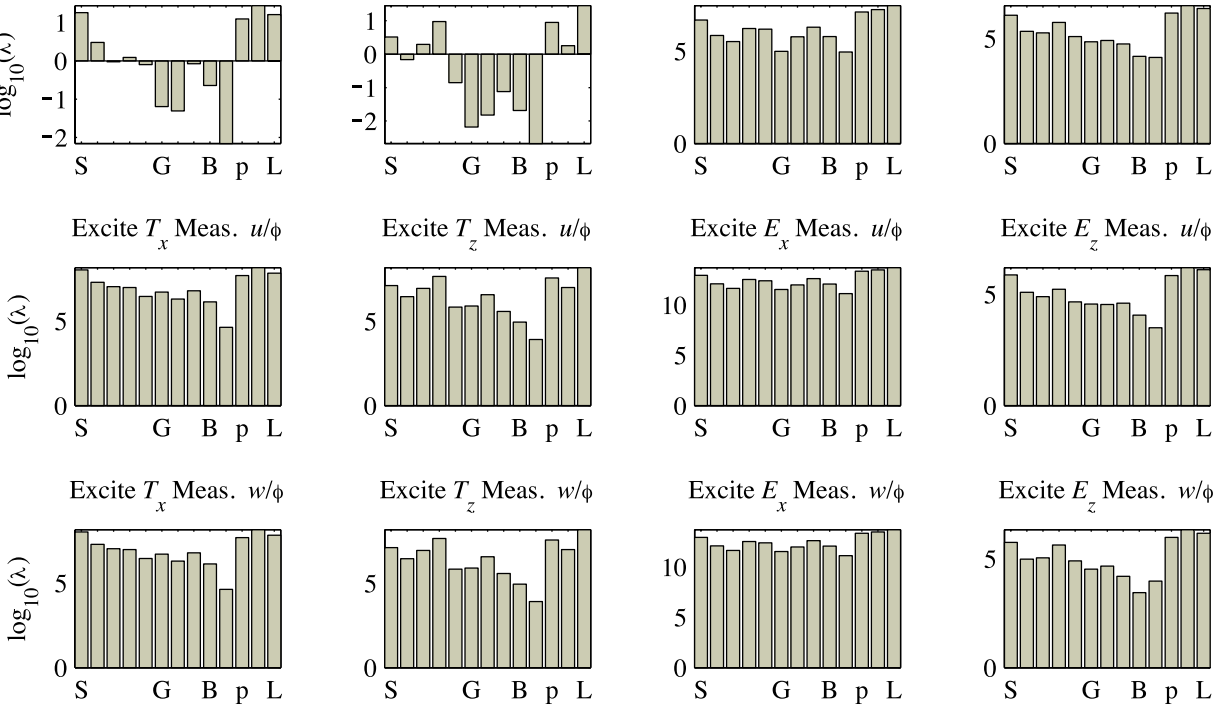

Excite $T_{x}$ Meas. $u / w / \phi$

Excite $T_{z}$ Meas. $u / w / \phi$

Excite $E_{x}$ Meas. $u / w / \phi$

Excite $E_{z}$ Meas. $u / \phi$

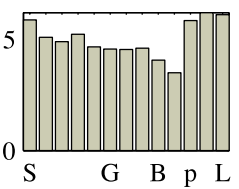

Excite $E_{z}$ Meas. $w / \phi$
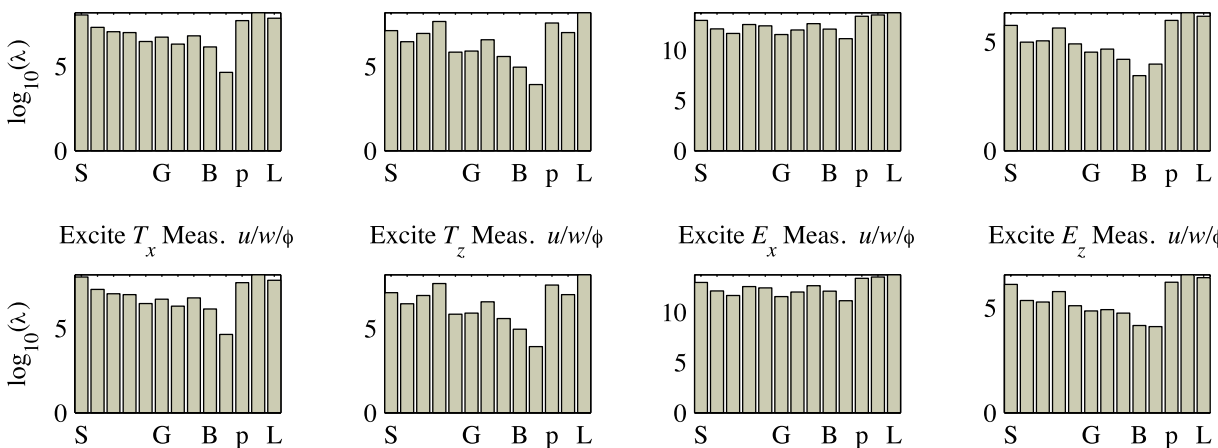

Excite $E_{z}$ Meas. $u / w / \phi$

Excite $T_{x}$ Meas. $\phi$
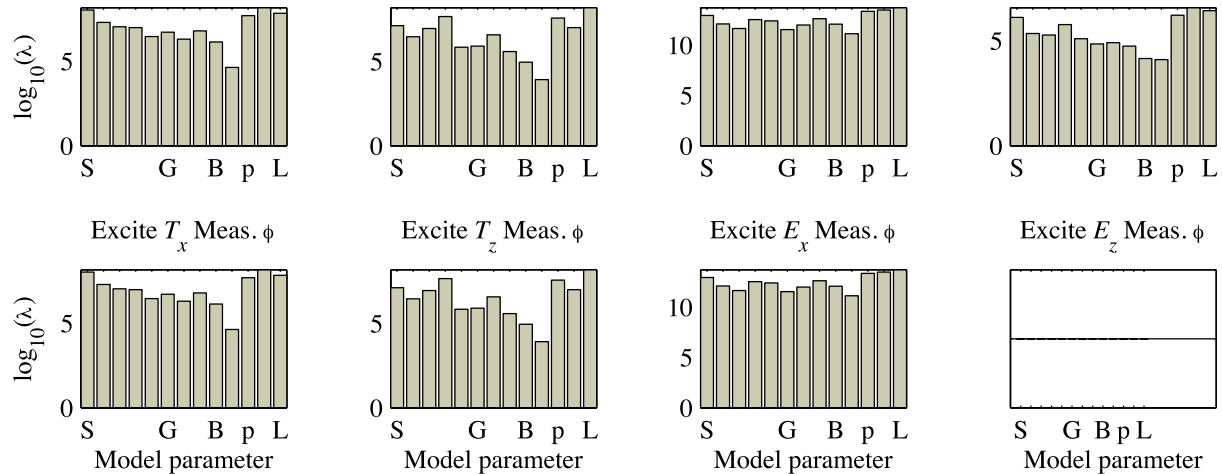

Excite $E_{z}$ Meas. $\phi$

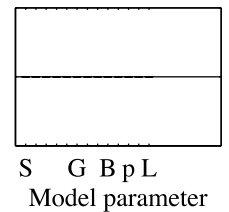

Figure 15: Calculated $\lambda$ sensitivity factors against model parameters 

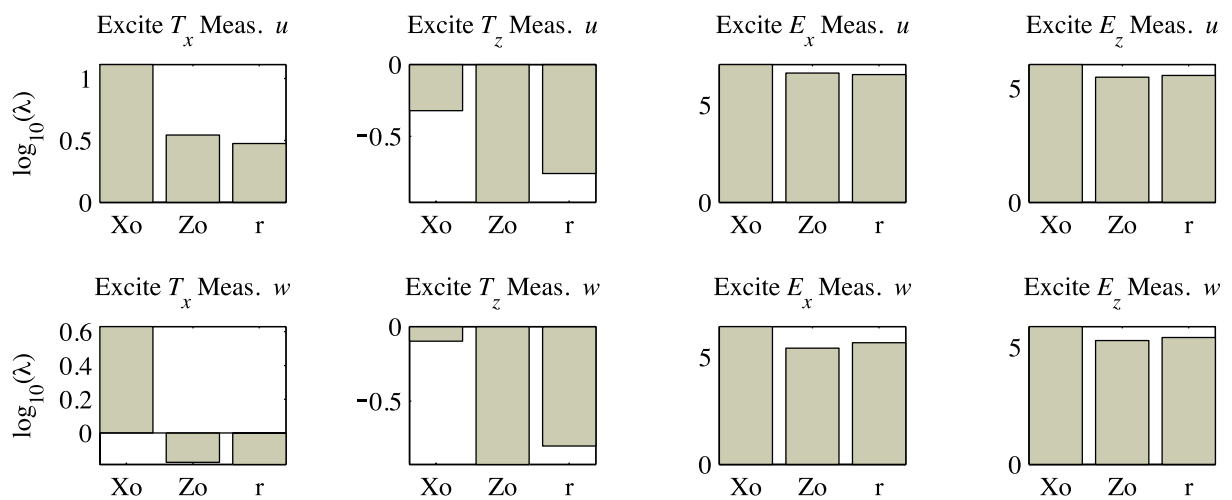

Excite $E_{x}$ Meas. $w$

Excite $E_{z}$ Meas. $w$
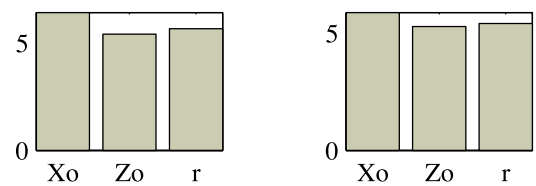

Excite $T_{x}$ Meas. $u / w$

Excite $T_{z}$ Meas. $u / w$

Excite $E_{x}$ Meas. $u / w$

Excite $E_{z}$ Meas. $u / w$
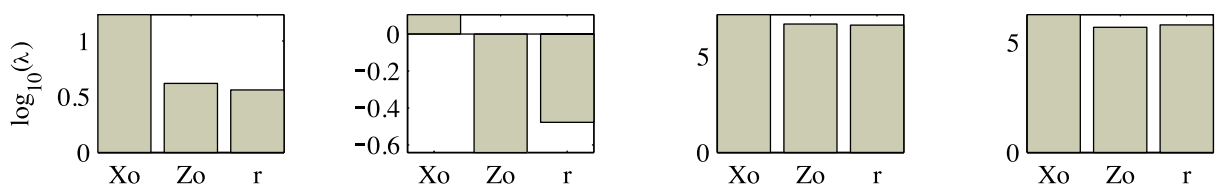

Excite $T_{x}$ Meas. $u / \phi$

Excite $T$ Meas. $u / \phi$

Excite $E_{x}$ Meas. $u / \phi$

Excite $E_{z}$ Meas. $u / \phi$
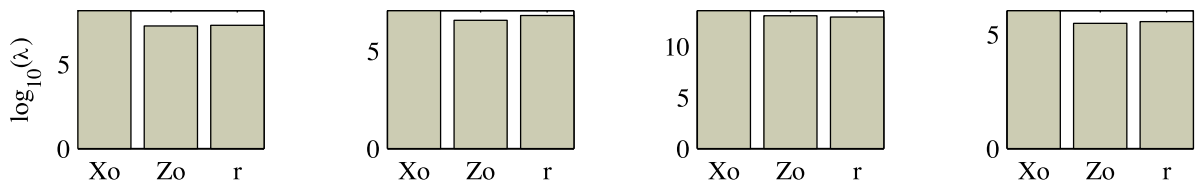

Excite $T_{x}$ Meas. $w / \phi$

Excite $T_{z}$ Meas. $w / \phi$

Excite $E_{x}$ Meas. $w / \phi$

Excite $E_{z}$ Meas. $w / \phi$
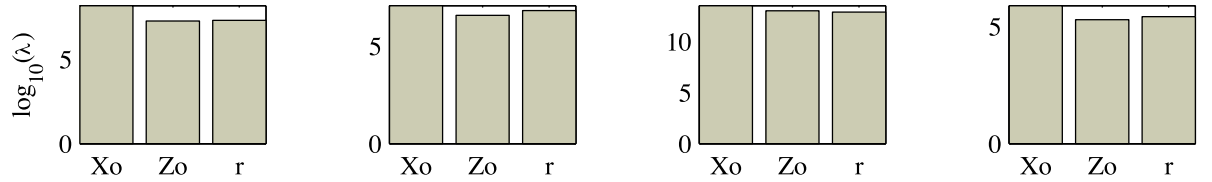

Excite $T_{x}$ Meas. $u / w / \phi$

Excite $T_{z}$ Meas. $u / w / \phi$

Excite $E_{x}$ Meas. $u / w / \phi$
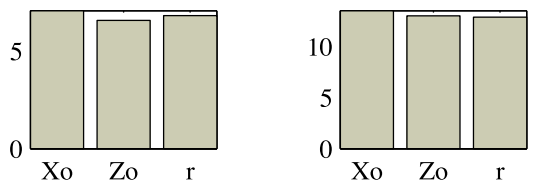

Excite $E_{z}$ Meas. $u / w / \phi$
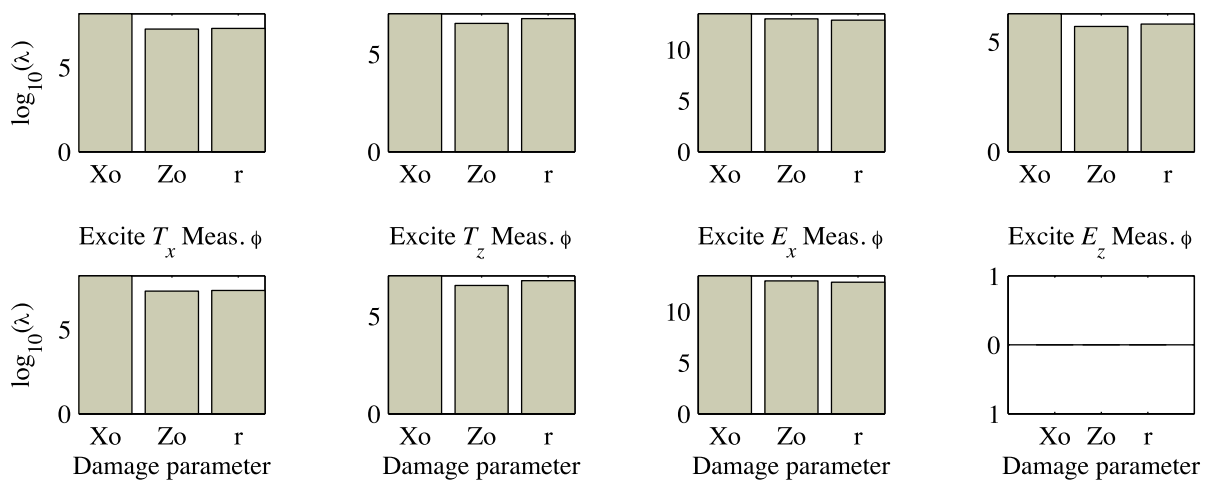

Figure 16: Calculated $\lambda$ sensitivity factors against damage parameters 

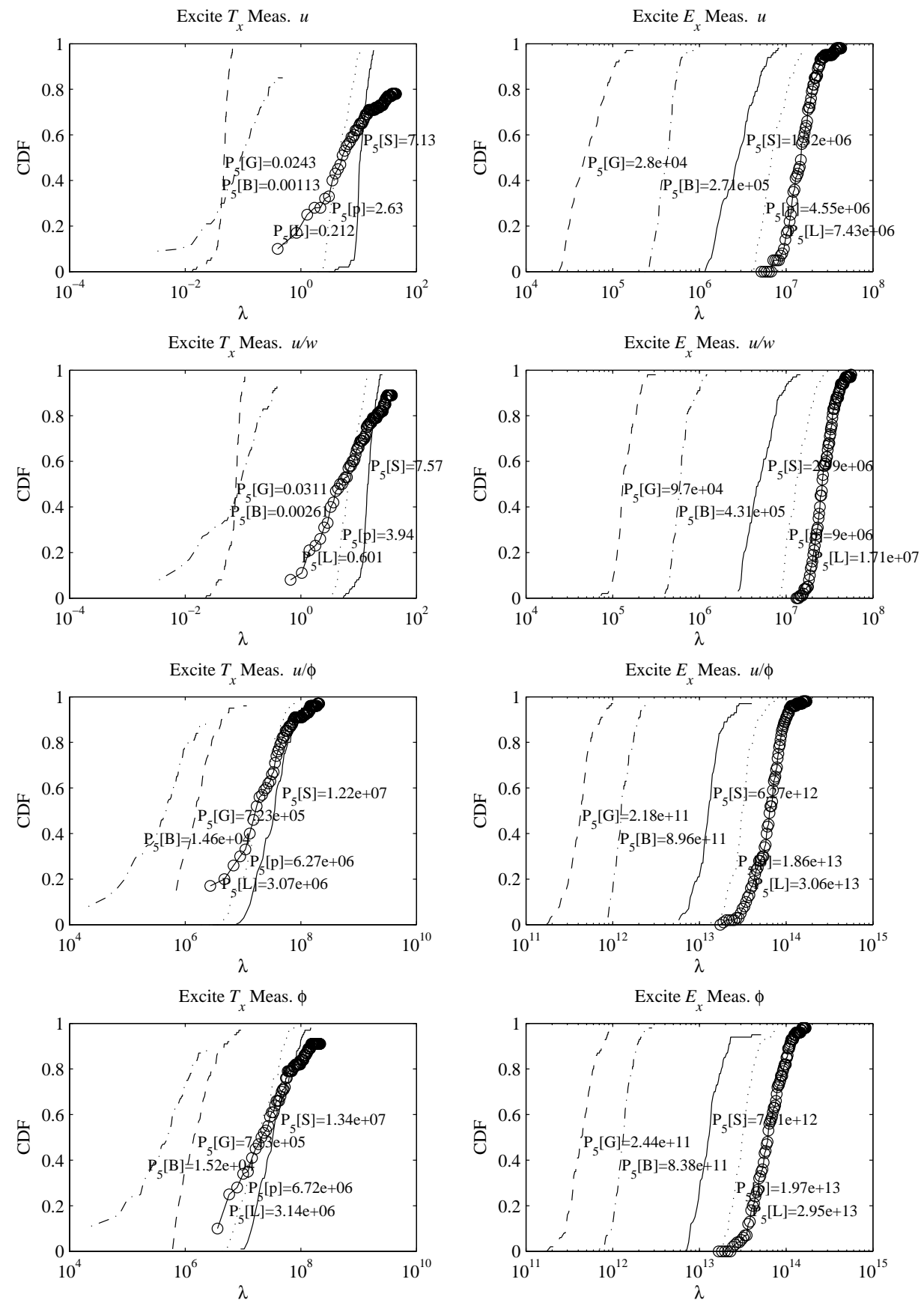

Figure 17: Density function of calculated $\lambda$ sensitivity factors against model parameters 

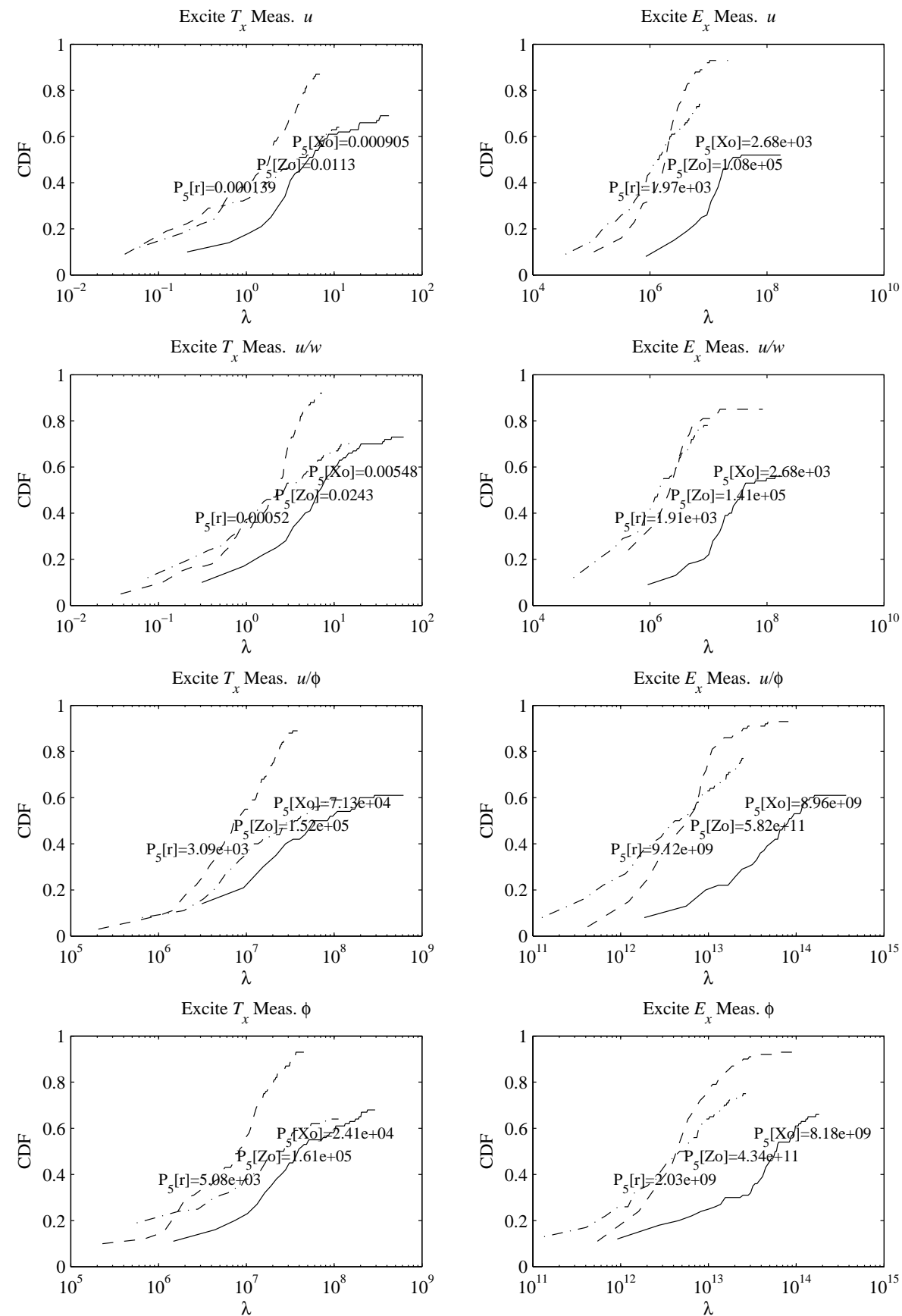

Figure 18: Density function of calculated $\lambda$ sensitivity factors against damage parameters 


$$
\Lambda^{-1}=\sum_{j} \lambda_{j}^{-1} \sum_{k} \lambda_{k}
$$

\begin{tabular}{l|rrrr} 
& $T_{x}$ & $T_{z}$ & $E_{x}$ & $E_{z}$ \\
\hline \hline$u$ & 43.4 & 314.9 & 31.5 & 32.7 \\
$v$ & 63.2 & $\mathbf{5 7 6 . 7}$ & $\mathbf{1 2 0 . 9}$ & $\mathbf{6 1 . 1}$ \\
$u \mid v$ & 46.7 & 446.6 & 38.1 & 44.1 \\
$u \mid \phi$ & 46.1 & 143.4 & 33.7 & 32.7 \\
$v \mid \phi$ & 47.3 & 151.3 & 37.9 & 61.1 \\
$u|v| \phi$ & 46.6 & $\mathbf{1 5 5 . 8}$ & 35.8 & 44.1 \\
$\phi$ & 46.8 & $\mathbf{1 3 8 . 5}$ & 34.8 & -
\end{tabular}

Table 3: Estimator $\Lambda$ between combined model sensitivity $\lambda_{j}$ and damage sensitivity $\lambda_{k}$ for each excitation/measurement setup.

Two conclusions are obtained. The first, geometrical properties $L_{x}, L_{z}$, density $\rho$ and the main elastic constant $s_{11}$ should be known with higher precision for most experimental setups, since their uncertainties maximally affect the cost functional. The second, horizontal position $x_{0}$ will be recovered with slightly higher precision than the remaining parameters, according to the $\lambda$ sensitivity factor. The intersection of both conclusions makes $E_{x}$ followed by $T_{z z}$ excitations with either voltage $\phi$ or normal displacement $v$ (and possibly their combination $u|w| \phi$ ) measurement the best monitoring system setup. The representative combinations with better identifiability are highlighted in Table 3.

\subsection{Excitation design}

The choice of excitation time dependency is defined by the excitation values: i) central frequency $f$, ii) bandwidth $b$ and iii) the time $T$ of excitation and measurement. The optimal design, in the sense of maximal sensitivity to damage parameters and minimal sensitivity to model uncertainties, is studied through the evolution computation of the respective $\lambda$ sensitivity factors.

Figure 19 shows the $\lambda$ sensitivity to model parameters (top) and to damage parameters (bottom) versus the excitation values. The choice $f=200$ $[\mathrm{kHz}], b=50 \% f$ and $T=30[\mu \mathrm{s}]$ is justified by the trade-off between high sensitivity to damage parameters and low sensitivity to model parameters. 

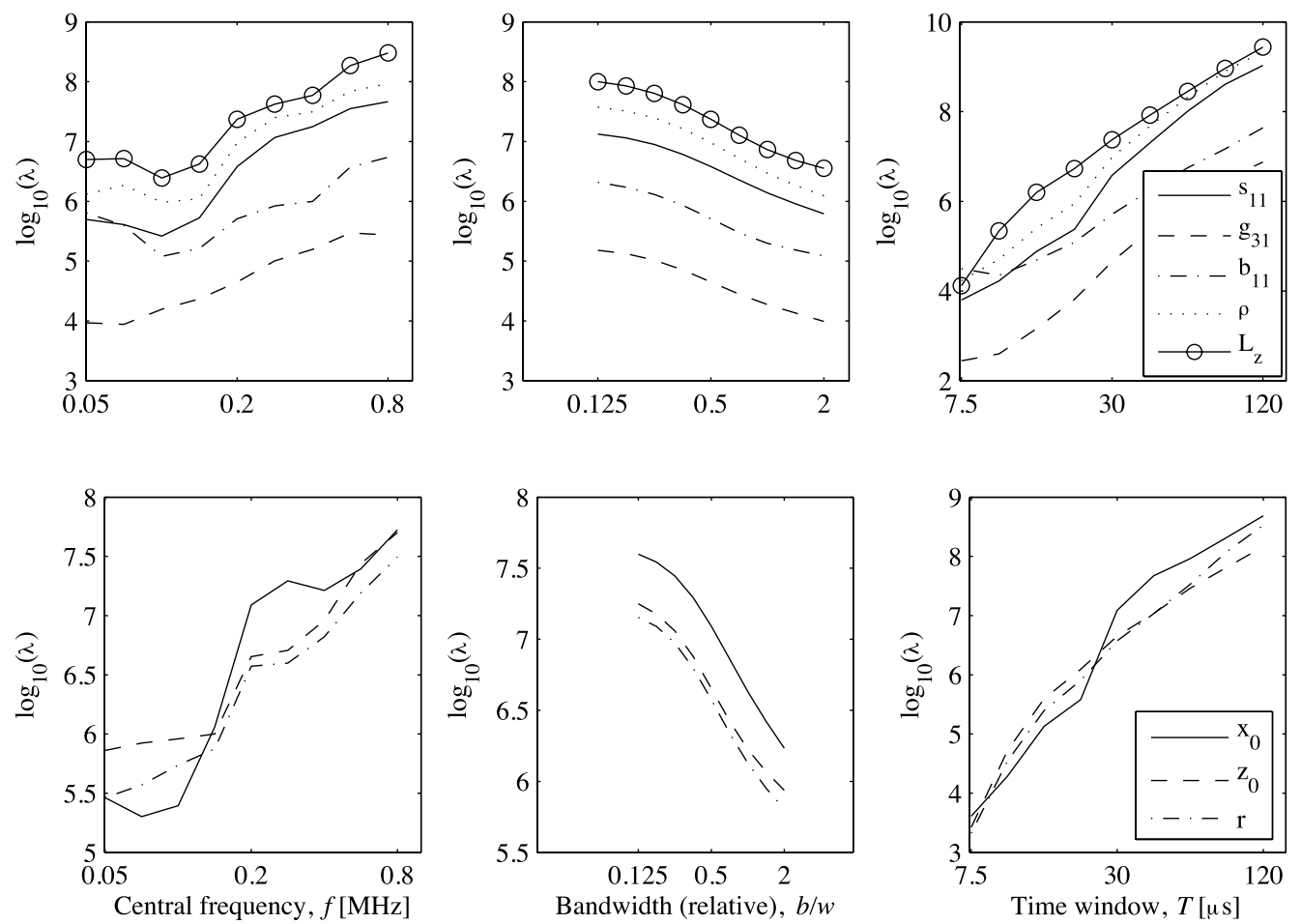

Figure 19: $\lambda$ sensitivity to model parameters (top) and to damage parameters (bottom) versus excitation values (ase of excitation $E_{x}$ and measurement $u$ )

Furthermore, this choice is supported by the following physical interpretation: the $T$ allows the fastest mechanical wave to travel and return along the specimen, carrying information about the defect to the measured surface. On the other hand, the $f$ value is physically supported by the fact that corresponds to a wavelength of the sample damage diameter order of magnitude, about $1 / 6$ of the specimen size. The chosen $b$ adopts a typical value used in standard ultrasonic probes.

\subsection{Error sources}

The final error in the recovered parameters is assumed to be a combination of the measurements error, of that of the mathematical model that simulates the experimental system, and of the inversion algorithm error. The objective of this section is to provide a quantifier of the latter.

The error in the inversion algorithm can be estimated by a statistical analysis of the reconstructed parameters imposing that the other two er- 

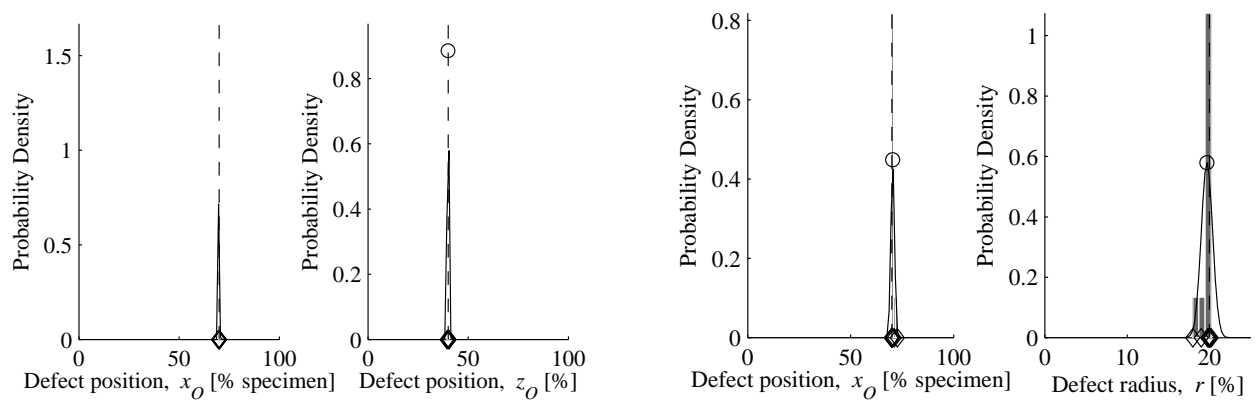

Search on parameters $\left[x_{0}, z_{0}\right]$
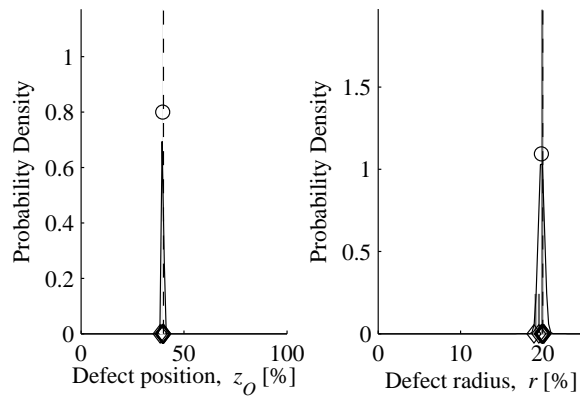

Search on parameters $\left[x_{0}, r\right]$
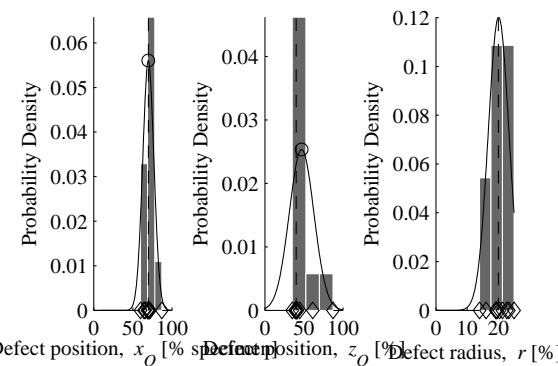

Search on parameters $\left[z_{0}, r\right]$

Search on parameters $\left[x_{0}, z_{0}, r\right]$

Figure 20: Statistical distribution of reconstructed parameters. First three figures: twoparameter search. Last figure: three-parameter search

ror sources are zero. This can be achieved using directly as experimental measurements those produced by the computational model, without adding neither simulated noise nor model uncertainties.

Figure 20 shows the statistical distribution of the working parameters for search cases with various combinations of parameters as unknown, leaving the remaining as known at the true values. The experimental setup adopted here is the case of a mechanical excitation at the right side and measurement of electric potential. The graphics include, in addition to the true value, the values obtained in ten realizations of the search, their histograms and the best fitting gaussian curve.

Also, Figure 21 shows the correlation between the final CF (i.e. for the reconstructed parameters) and the standard deviation of the material constants within ten realizations of the search. 

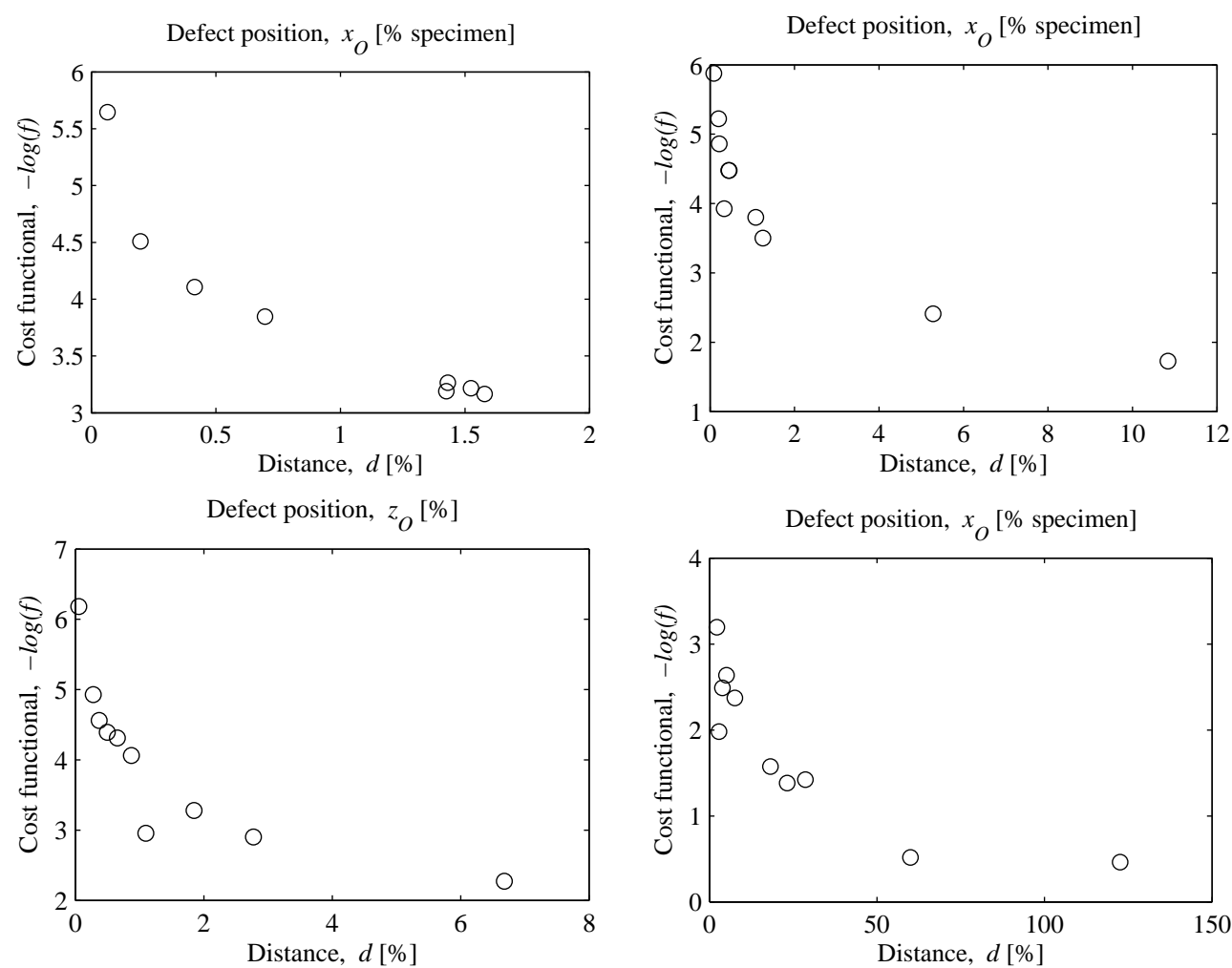

Figure 21: Reconstructed parameters versus cost functional. First three figures: twoparameter search. Last figure: three-parameter search 


\subsection{Effect on working parameters}

The influence of the partial results from the reconstructed defect error shown above is verified in Figures 22 to 25, that plot distance between real and reconstructed damage parameters, versus system uncertainty levels.

These relationships are computed for selected excitation/measurement configurations, and for several combinations of working parameters, with the aim of quantifying the final identifiability of damage parameters in relationship with the experimental configuration. The distance in the reconstructed damage is estimated by its mean and standard deviation for three realizations of model parameters and full GA searches for each realization. The prohibitive computational cost of this nested set of computations fully justifies the need for the time-domain sensitivity factor $\lambda$ presented in this paper. Figure 26 shows that the sensitivity estimator $\Lambda$ allows to successfully differentiate the experimental setups with best distance to uncertainty ratio.

These figures corroborate the fact that $T_{z z}$ followed by $E_{x}$ excitations with either normal displacement $v$ or potential $\phi$ measurements (and possibly their combination $u|w| \phi)$ are the best monitoring system setups. The choice of the best single setup depends on the precision attained in each excitation and measurement. For instance, simpler and higher precision measurement of potential $\phi$ than displacement $v$ may compensate a lower $\Lambda$ for the overall error.

Simulating uncertainties directly as a gaussian noise applied to the measurements is recently becoming standard practice in inverse problem research. But no proper justification of their magnitude or probability distribution exists. Therefore, the analysis of uncertainties introduced in the model is compared in Figure 27 to an equivalent analysis with uncertainties introduced directly on the measurements. This is to verify whether the trends are consistent.

The assumption that implies that noise can be simulated in a gaussian distribution over measurements appears not to be fully consistent with noise originated from uncertainties in the model, in the sense that their distribution is not independent. Hence, the effect of noise on the number of measurements is not captured correctly by the simplification (see the difference from the measurement of $u$ with those of $u|w| \phi$, in Figure 22 and Fig. 27.). 

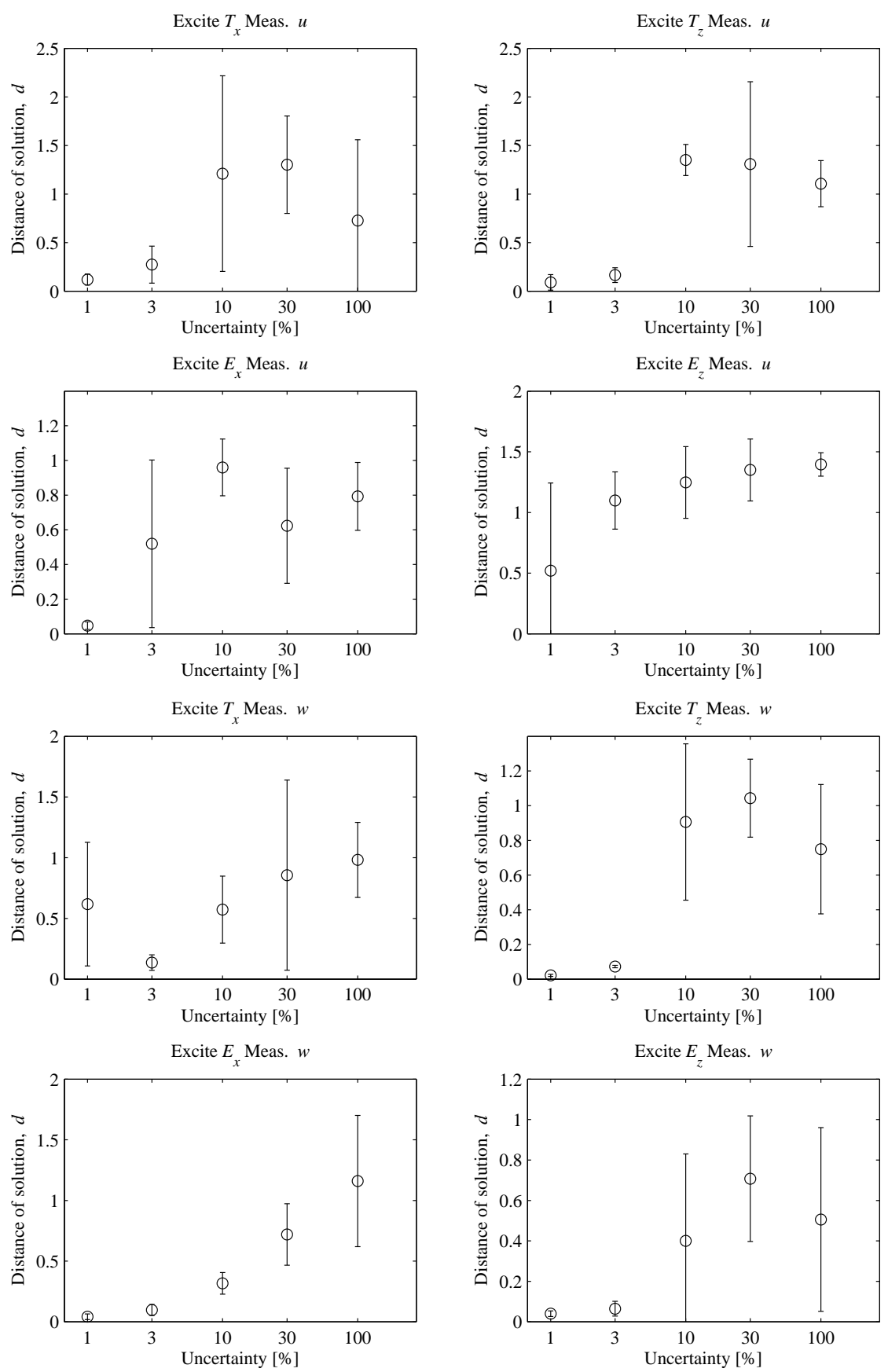

Figure 22: Damage distance versus model uncertainties (1 of 4) 

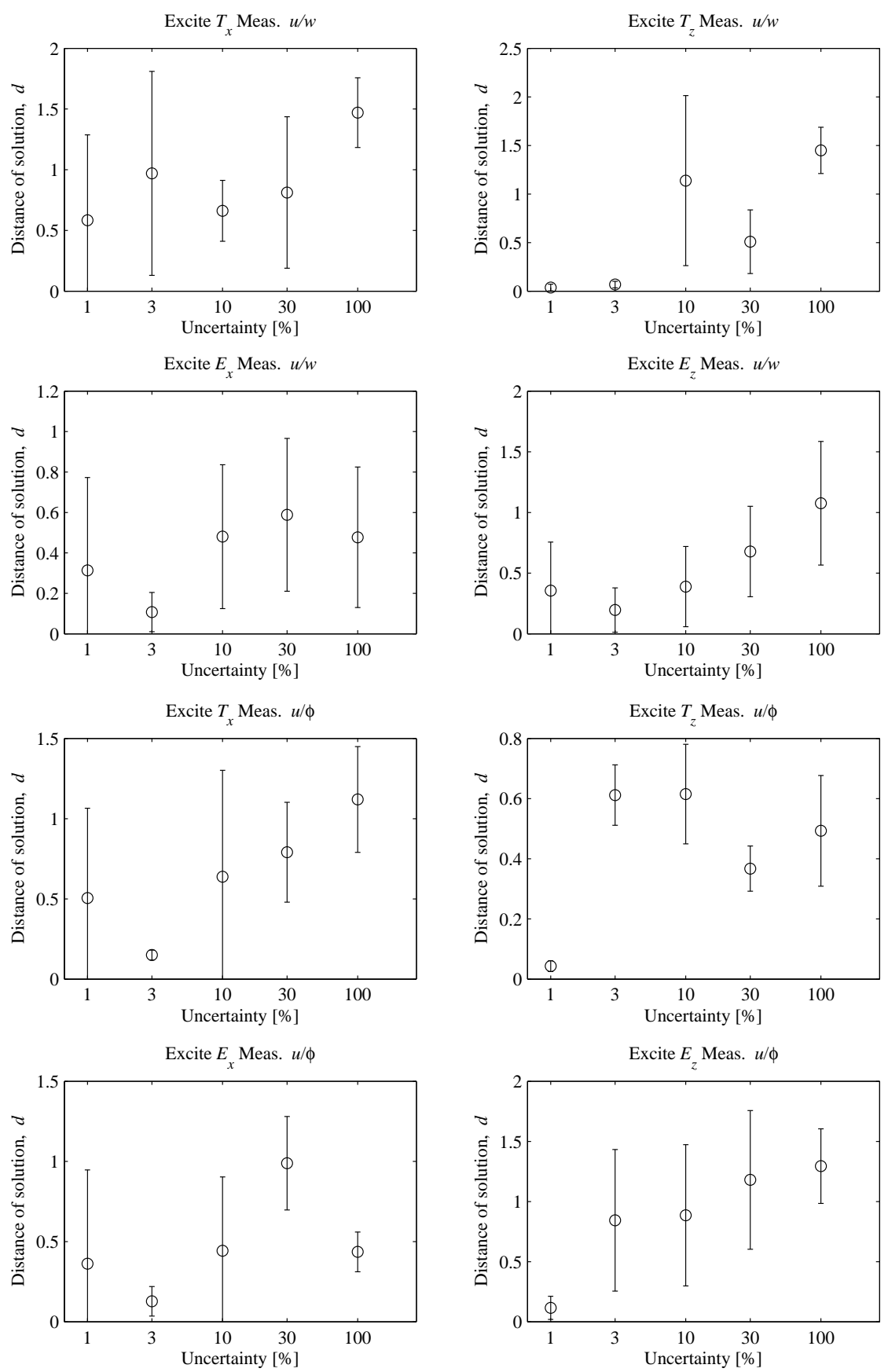

Figure 23: Damage distance versus model uncertainties (2 of 4) 

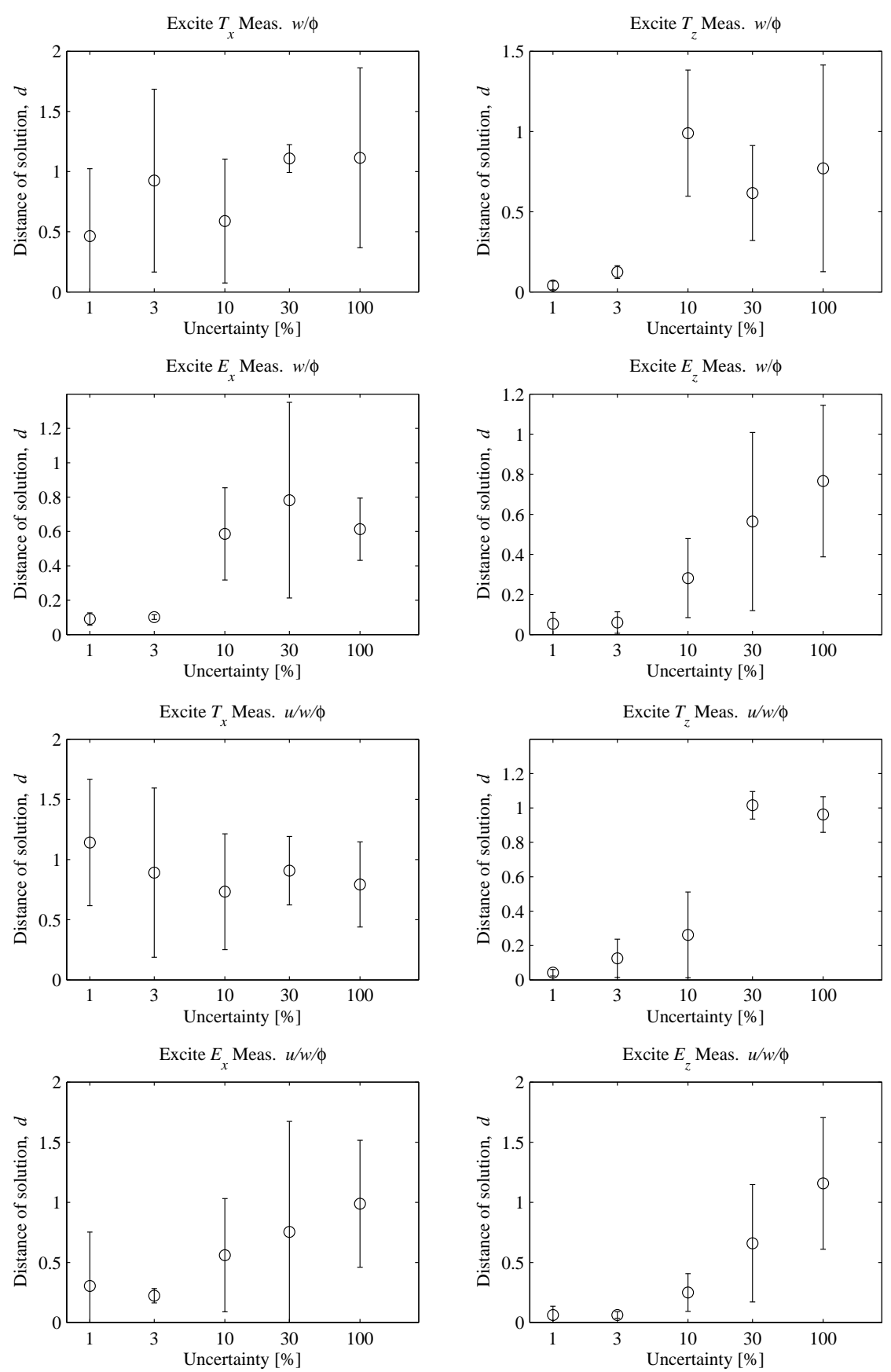

Figure 24: Damage distance versus model uncertainties (3 of 4) 

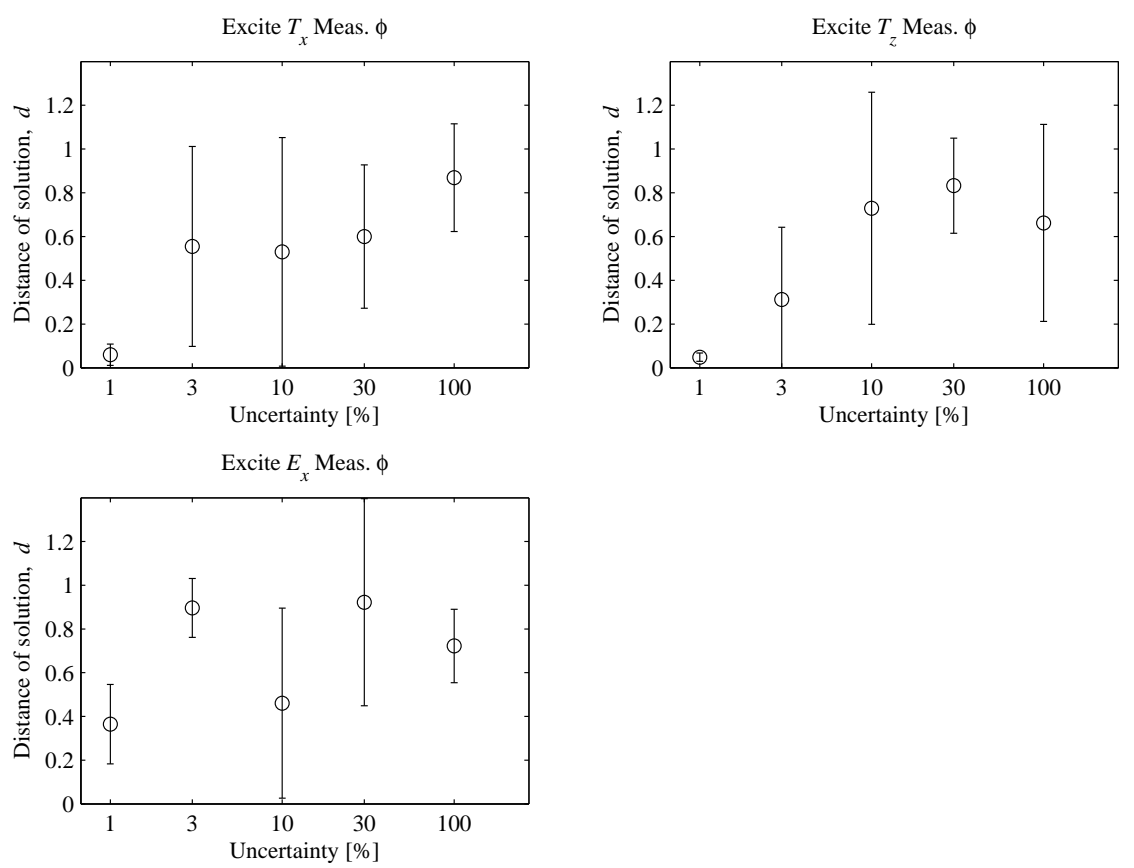

Figure 25: Damage distance versus model uncertainties (4 of 4)

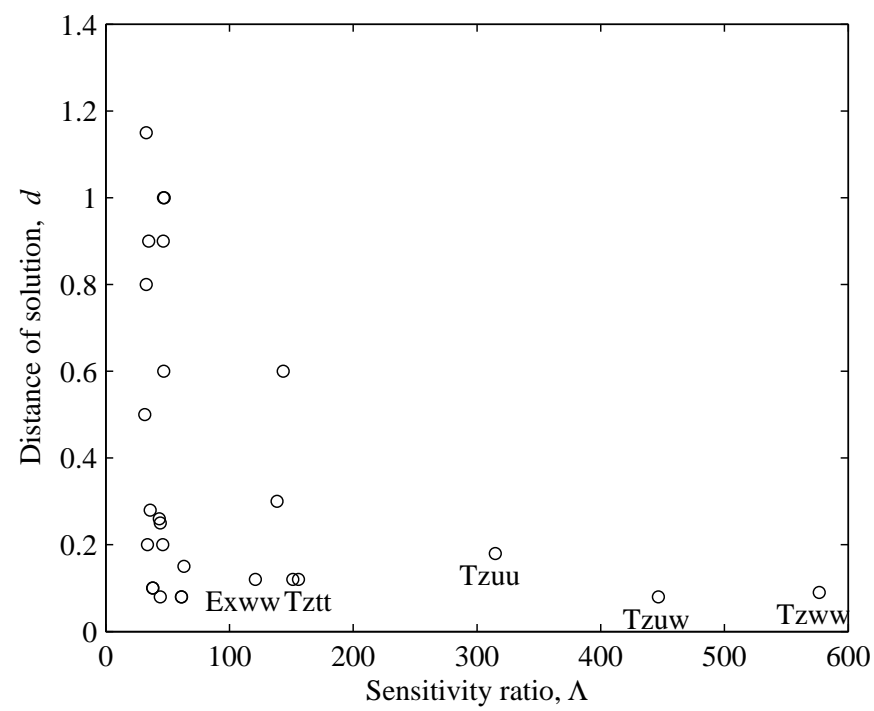

Figure 26: Correlation between sensitivity estimator $\Lambda$ and the average solution distance for $3 \%$ model uncertainty. 

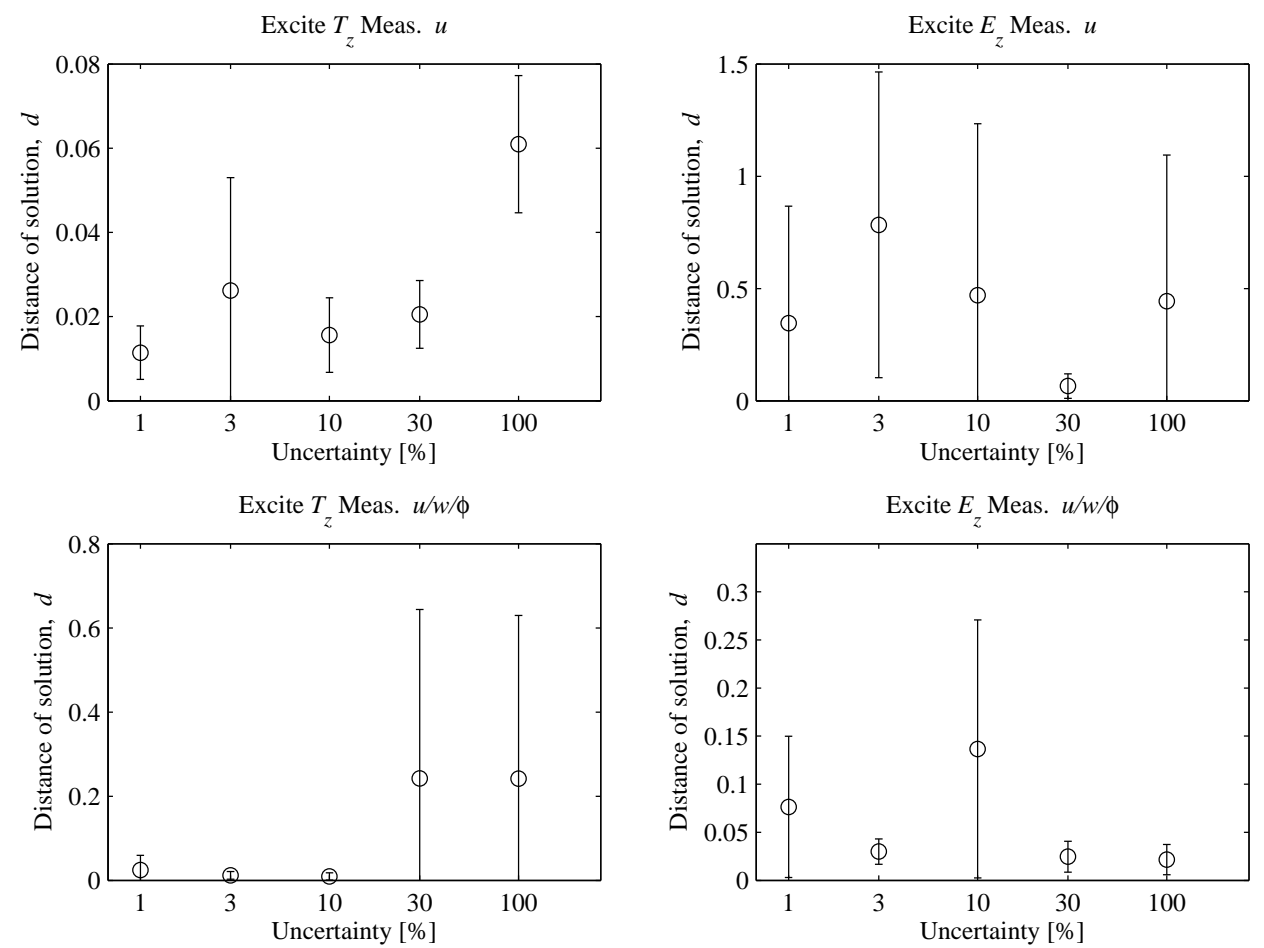

Figure 27: Distance of the damage versus noise in measurements, for the search of $\left[x_{0} y_{0}\right.$ $r]$. 


\section{Discussion}

An inverse problem strategy is defined and tested, capable of identifying and characterizing a cavity-type defect in a piezoelectric plate. Model parameter uncertainties as well as measurement noise are present. To quantify the level of certainty of every parameter, a sensitivity analysis is developed, introducing and implementing a factor $\lambda$. This indicator quantifies the damage parameters most sensitive to both uncertainties and noise. The sensitivity analysis also permits the determination of the model parameters that require higher precision to control the reconstruction error.

In order to design a robust monitoring technique, the choice effect on the excitation/measuring type is studied based on the sensitivity factors. From the point of view of uncertainty, the electrical excitation transverse to the polarization direction (either with normal displacement or potential measurements), is identified as the best monitoring system setup. These setups combine a moderate sensitivity to uncertainty in model parameters.

It is concluded that, by applying the proposed modified model-based inverse problem strategy, it is possible to reconstruct the defect characteristics with sufficient precision, under realistic levels of measurements noise and in the presence of uncertainties in the specimen model.

\section{Acknowledgements}

The authors would like to thank the Ministerio de Innovación y Ciencia, Spain, for the FPU grant AP-2006-02372 and also from grants Excelencia Junta Andalucía P08-TEP-3641, MCyT DPI 2002-04472-C02-02. Authors would also like to thank Prof. Rafael Gallego for his invaluable contributions to the development of the present work.

\section{References}

Allik, H., Hughes, T., 1970. Finite elment method for piezoelectric vibration. International Journal for Numerical Methods in Engineering 2, 151-157.

Dennis, J.E. and Schnabel R.B. , 1983. Numerical Methods for Unconstrained Optimization and Nonlinear Equations. SIAM.

Gallego, R., Rus, G., 2004. Identification of cracks and cavities using the topological sensitivity boundary integral equation 33 . 
Goldberg, D. E., 1989. Genetic Algorithms in search, Optimization and Machine Learning. Addison-Wesley.

Kaltenbacher, B., Lahmer, T., Mohr, M., Kaltenbacher, M., 2006. Pde based determination of piezoelectric material tensors. Europ. J. App. Mathem. $17,383-416$.

Kuna, M., 2010. Fracture mechanics of piezoelectric materials - where are we right now? Engineering Fracture Mechanics 77, 309-326.

Landis, C., 2002. A new finite-element formulation for electromechanical boundary value problems. International Journal for Numerical Methods in Engineering 55, 613-628.

Ou, Z. C., Chen, Y. H., 2003. Discussion of the crack face electric boundary condition in piezoelectric fracture mechanics. Int. J. of Fracture 123, L151L155.

Palma, R., Rus, G., Gallego, R., 2009. Probabilistic inverse problem and system uncertainties for damage detection in piezoelectrics. Mechanics of Materials 41, 1000-1016.

Pérez-Aparicio, J., Sosa, H., Palma, R., 2007. Numerical investigation of field-defect interactions in piezoelectric ceramics. International Journal of Solids and Structures 44, 4892-4908.

Ruíz, A., Ramos, A., San-Emeterio, J., 2004. Evaluation of piezoelectric resonator parameters using an artificial intelligence technique. Integrated Ferroelectric 63, 137-141.

Rus, G., Palma, R., Pérez-Aparicio, J., 2009. Optimal measurement setup for damage detection in piezoelectric plates. International Journal of Engineering Science 47, 554-572.

Schneider, G., 2007. Influence of electric field an mechanical stresses on the fracture of ferroelectrics. Annu. Rev. Mater. Res. 37, 491-538.

Tarantola, A., 2005. Inverse Problem Theory. SIAM.

Taylor, R. L., 2003. Feap - a finite element analysis program. version 8.2, rlt@cs.berkeley.edu. 
Zienkiewicz, O., Taylor, R., Zhu, J., 2005. The Finite Element Method: The Basis. Elsevier Butterworth-Heinemann. 